\title{
Simulations and flight tests of a new nonlinear controller for the EAGLE lander
}

\author{
Marco Sagliano * $\quad$ Michael Dumke ${ }^{\dagger}$ \\ Stephan Theil $\ddagger$ \\ German Aerospace Center, Robert Hooke Straße 7, Bremen, Germany, 28359
}

This paper describes the complete control strategy developed for EAGLE, a Vertical Take-off, VerticalLanding vehicle conceived and realized by the German Aerospace Center for the testing and the validation of GNC technologies. A nonlinear controller, divided in thrust-vector control and position control, based on Sliding Mode Theory, has been developed. Simulations and real flight tests, realized in a DLR ad-hoc facility, are described. Results show that the proposed control strategy is able to successfully control EAGLE in presence of a highly constrained scenario, as well as multiple uncertainties and disturbances.

\section{Nomenclature}

$\begin{array}{ll}\text { Acronyms } & \\ \text { CoM } & =\text { Center of Mass } \\ \text { CoP } & =\text { Environment for Autonomous GNC Landing Experiments } \\ \text { EAGLE } & =\text { Degrees of Freedom } \\ \text { DOF } & =\text { Electronic Control Unit } \\ \text { ECU } & =\text { Engine Power Settings } \\ \text { EPS } & =\text { Guidance, Navigation, and Control } \\ \text { GNC } & =\text { Global Navigation Satellite System } \\ \text { GNSS } & =\text { Inertial Measurement Unit } \\ \text { GUI } & =\text { Lunar Landing Training Vehicle } \\ \text { IMU } & =\text { Lunar Landing Vehicle } \\ \text { LLTV } & =\text { Onboard Computer } \\ \text { LLV } & =\text { Sliding Mode Control } \\ \text { OBC } & =\text { Telemetry / Telecommand } \\ \text { RC } & =\text { Thrust Vector Control } \\ \text { SMC } & =\text { Vertical Take-off, Vertical Landing } \\ \text { TM/TC } & \end{array}$

Roman

$\begin{array}{ll}\mathbf{A} & =\text { Affine vector } \\ \mathbf{A}_{\theta} & =\text { affine term for the Euler angle, } \mathrm{rad} \mathrm{s}^{-2} \\ A_{x} & =\text { affine term for the altitude, } \mathrm{m} \mathrm{s}^{-2} \\ a_{i} & =\mathrm{i}^{\text {th }} \text { thrust coefficient, } \mathrm{N} \\ a_{V} & =\text { Voltage coefficient, } \mathrm{V} \\ \mathbf{B} & =\text { Affine matrix } \\ \mathbf{B}_{\theta} & =\text { affine term for the Euler angle, } \mathrm{kg}^{-1} \mathrm{~m}^{-2} \\ B_{x} & =\text { affine term for the altitude, } \mathrm{kg}^{-1} \\ b_{V} & =\text { Voltage coefficient, } \mathrm{V} \\ \widehat{D} & =\text { Down unit vector }\end{array}$

*Research Engineer, Guidance, Navigation and Control Department, AIAA Member

${ }^{\dagger}$ Research Engineer, Guidance, Navigation and Control Department

${ }^{\ddagger}$ Department Head, Guidance, Navigation and Control Department

$$
1 \text { of } 23
$$




\begin{tabular}{|c|c|c|}
\hline$\widehat{E}$ & $=$ & East unit vector \\
\hline $\mathbf{e}_{\phi}$ & $=$ & Rotation vector \\
\hline $\mathbf{e}_{x, \mathrm{~B}}$ & $=$ & Unit vector in $\mathrm{B}$ reference frame \\
\hline f & $=$ & force, $\mathrm{N}$ \\
\hline $\mathbf{f}_{t h r}$ & $=$ & Thrust magnitude, $\mathrm{N}$ \\
\hline g & $=$ & $x$ Gravity acceleration vector, $\mathrm{m} \mathrm{s}^{-2}$ \\
\hline$g_{x}$ & $=$ & $x$ component of gravity acceleration, $\mathrm{m} \mathrm{s}^{-2}$ \\
\hline$I_{n}$ & $=$ & Identity matrix of dimensions $n$ \\
\hline I & $=$ & Inertia, $\mathrm{kg} \mathrm{m}^{2}$ \\
\hline$i, j, k, m, n$ & $=$ & Non-negative, integer indices \\
\hline $\mathbf{K}$ & $=$ & Adaptive gain, $\mathrm{N} \mathrm{m} \mathrm{s} \mathrm{rad}-1$ \\
\hline $\mathbf{K}$ & $=$ & Nonlinear-gain vector \\
\hline$\widehat{\mathbf{K}}$ & $=$ & Nonlinear-gain vector \\
\hline$k_{p}$ & $=$ & $\mathrm{p}^{\text {th }}$ nonlinear gains \\
\hline $\mathbf{l}_{\mathrm{TVC}}$ & $=$ & Level arm in TVC reference frame, $\mathrm{m}$ \\
\hline$m$ & $=$ & Mass, $\mathrm{kg}$ \\
\hline$\widehat{N}$ & $=$ & North unit vector \\
\hline$O_{n_{1} \times n_{2}}$ & $=$ & Zero matrix of dimensions $n_{1}, n_{2}$ \\
\hline q & $=$ & Quaternion \\
\hline $\mathbf{r}$ & $=$ & Position vector, $\mathrm{m}$ \\
\hline$s$ & $=$ & Sliding surface \\
\hline $\mathbf{t}$ & $=$ & torque, $\mathrm{N} \mathrm{m}$ \\
\hline $\mathbf{u}$ & $=$ & Control vector \\
\hline$V$ & $=$ & Pump Voltage, V \\
\hline$V$ & $=$ & Lyapunov function \\
\hline $\mathbf{v}$ & $=$ & velocity vector, $\mathrm{m} \mathrm{s}^{-1}$ \\
\hline$v_{0}$ & $=$ & First vane command, ms \\
\hline$v_{1}$ & $=$ & Second vane command, ms \\
\hline
\end{tabular}

$\begin{array}{lll}\text { Greek } & = & \text { Sliding-mode control layer } \\ \delta & = & \text { Linear-gain vector } \\ \Lambda & = & \text { Linear-gain vector } \\ \widehat{\Lambda} & = & \mathrm{p}^{\text {th }} \text { linear gains } \\ \lambda_{p} & = & \text { Rotation angle, } \mathrm{rad} \\ \phi & = & \text { Euler angle, } \mathrm{rad} \\ \theta & = & \text { Angular rate, } \mathrm{rad} \mathrm{s}^{-1} \\ \omega & \end{array}$

Operators and subscripts

$\operatorname{det}(\cdot)$

$\operatorname{sign}(\cdot)$

sat $(\cdot)$

$(\cdot)$

(.)

$(\cdot)_{\text {adap }}$

$(\cdot)_{\text {alt }}$

$(\cdot)_{\mathrm{B}}$

$(\cdot)_{\text {cmd }}$

$(\cdot)_{\text {meas }}$

$(\cdot)_{\mathrm{M}}$

$(\cdot)_{\mathrm{NED}}$

$(\cdot)_{\text {nom }}$

$(\cdot)_{p}$

$(\cdot)_{R C}$

$(\cdot)_{\text {real }}$

$(\cdot)_{\text {ref }}$

$(\cdot)_{t h r}$

$(\cdot)_{t o t}$

$(\cdot)_{\mathrm{TVC}}$
$=$ Determinant of a matrix

$=$ Sign function

$=$ Saturation function

$=$ First-time derivative, $[\cdot] \mathrm{s}^{-1}$

$=$ Second-time derivative, $[\cdot] \mathrm{s}^{-2}$

$=$ Adaptive

$=$ Altitude component

$=\mathrm{B}$ reference frame

$=$ Commanded

$=$ Measured

$=$ Mechanical reference frame

$=$ NED reference frame

$=$ Nominal

$=p^{\mathrm{th}}$ component of vector $(\cdot)$

$=$ Roll control

$=$ Real

$=$ Reference

$=$ Thrust

$=$ Total

$=\quad \mathrm{TVC}$ reference frame 


\section{Introduction}

Recent and future missions are targeting a precise descent and landing. This can be for instance the landing powered descent and landing of a re-usable first stage of a launch vehicle as it was demonstrated several times by SpaceX with its Falcon 9 launch system. On the other hand precise descent and landing has been applied for planetary landings and is foreseen for many more future missions to Mars and Moon. The development of Guidance, Navigation, and Control (GNC) techniques for these applications remains a challenging task although several mission have been already successfully completed. Especially in Europe the experience in this field of GNC is worth an improvement.

Several ideas to support and accelerate the GNC development using demonstrators have been conceived in the past in Europe. Only a very few have been implemented like the HOMER demonstrator of Airbus Defense and Space 112 2 Many of these ideas got stuck due to the high cost and high complexity of an experimental vehicle using rocket engines for the main thrust. The issue of handling explosive or toxic materials in an experimental vehicle creates high costs and limits flexibility and turn-around time. In order to decouple the high complexity of rocket propulsion from the GNC development the experimental Vertical Take-Off and Landing (VTOL) vehicle EAGLE (Environment for Autonomous GNC Landing Experiments) was conceived. The idea is based on NASA's Lunar Landing Training Vehicle (LLTV) of the Apollo program which was used to train the pilots of the lunar landing vehicles (LLV).
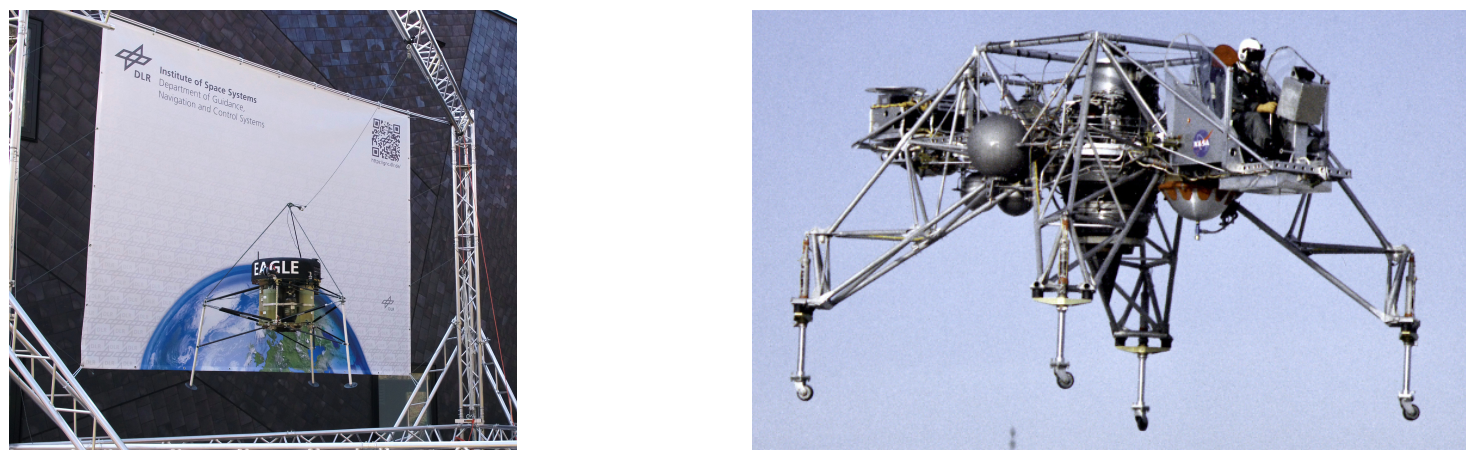

Figure 1: The experimental VTOL vehicle EAGLE (left) and its ancestor NASA's Lunar Landing Training Vehicle (LLTV) of the Apollo program ${ }^{\text {a }}$ (right).

The main goal of EAGLE is to provide dynamics, sensors and actuators similar to a landing vehicle. For most applications so far this included a vertical main thruster with thrust vector control Thrust Vector Control (TVC) and a Reaction Control System (RCS). Compared to the LLTV there is one major difference. The LLTV was designed to create environment and dynamics similar to the lunar landing environment. For that reason it was using a part of the main thrust for gravity compensation and the vehicle attitude was decoupled from the main thrust direction. This way it was achieved that the pilot's "feeling" when flying the LLTV was very close to the real LLV on the Moon. Since the goal of EAGLE is to "train" GNC software the gravity compensation can be omitted due to the fact that a different gravity level can easily be incorporated in the GNC software and there is no human-in-the-loop to be considered.

Based on the requirements for simple handling, similar flight dynamics, high flexibility, and fast turnaround times a small VTOL vehicle with a jet engine as main thruster was developed.

One of the critical subsystems of such a lander is represented by the control. The system has to be able to steer the spacecraft as predicted while being in an environment where multiple uncertainties and disturbances can act. Identification activities can be carried out for reducing the uncertainties associated with the spacecraft itself, but of course they cannot usually be reduced to zero. Similar conclusions can be drawn for the disturbances. Despite the efforts to develop and implement more accurate models (e.g., for characterizing the gravitational field) there will be disturbances, which must be rejected by the control subsystem without being known a priori (e.g., for bodies with an atmosphere like the Earth or Mars wind gusts can generate significant disturbances acting on the system). Finally, in the general case largely offnominal trajectories to satisfy the mission requirements might be needed. This means that linear controllers, designed around specific conditions, could experience a significant worsening of performance, or even worse,

asource: Great Images in NASA; Image \#: ECN-506; Date: 01/01/1964 
might no longer guarantee the stability of the system. This aspect can be mitigated by following a gainscheduling approach. However, this method, although working for several cases, suffers from a curse of dimensionality in case complex trajectories might be demanded. Moreover, there is no formal proof of the stability of the system between gain-scheduled controllers, for which usually a linear interpolation technique is employed. These aspects, together with the continuous enhancement of modern control techniques, suggest that an intrinsic nonlinear strategy for EAGLE could be employed. Specifically, sliding-mode control ${ }^{3} \sqrt{5}$ provides a straightforward framework for designing Lyapunov-stable, nonlinear controllers, able to deal with off-nominal conditions, and at the same time to provide excellent performance. This class of controllers has already been studied for several applications, including longitudinal flight control,$[6$ asteroid descent guidance ${ }^{7}$ and hypersonic atmospheric entry $\sqrt[8]{9}$ In this work the application of a 6 -DOF sliding-mode control technique for EAGLE will be described.

This paper is organized as follows: Sec. III provides an overview of the vehicle, while Sec. III will describe some of the identification activities carried over. The control strategy is described in Sec. IV] while simulation and flight-test results will be the subject of Secs. V] and VI, respectively. Finally, some conclusions are drawn in Sec. VII together with the further steps and developments foreseen for EAGLE.

\section{EAGLE system overview}

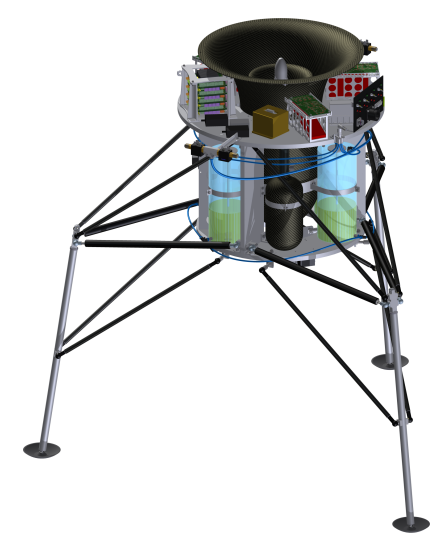

(a) EAGLE rendered in the current configuration

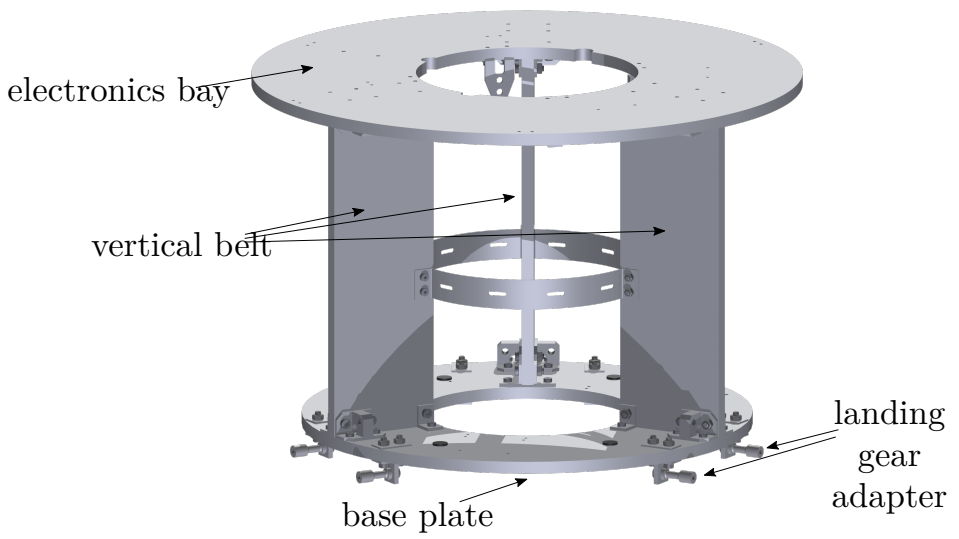

(b) Light weight main sandwich structure of EAGLE

Figure 2: (a) rendered model and (b) main structure of EAGLE.

The most apparent features of EAGLE, see Fig. 2a, are the landing legs and the main structure centrally housing the main engine. The main engine is a one stage jet engine capable of lifting the roughly $30 \mathrm{~kg}$ wet-mass of the lander. The landing gear absorbs the landing shocks protecting the sensitive electronics, payloads, and the structure itself. The light-weight main structure of EAGLE is build from five aluminum sandwich parts interconnected with aluminum brackets and clamps. Figure $2 \mathrm{~b}$ shows the two rings that are linked with three (identical) vertical belts. The upper ring is the electronics bay housing the overall avionics of the lander, the base plate closes the middle section for the fuel and gas tanks.

The central cutaway is reserved for the main engine and the TVC system deflecting the exhaust stream of the turbine. Figure 3 shows the landing gear system in fully extended and fully retracted configuration. Each leg is connected with four carbon rods supported with joints on each side. Additionally, each leg is hold by two gas dampers in the extended position (Fig. 3 (left)) in order to absorb shocks and forces during landing (Fig. 3 (right)).

\section{A. Actuation}

Three actuation systems are integrated within the lander. First of all, the jet engine generating the main thrust by commanding the fuel flow into the jet engine's burning chamber. Second, a Thrust Vector Control system employing two vanes aligned perpendicular to each other to deflect the thrust for pitch and yaw 

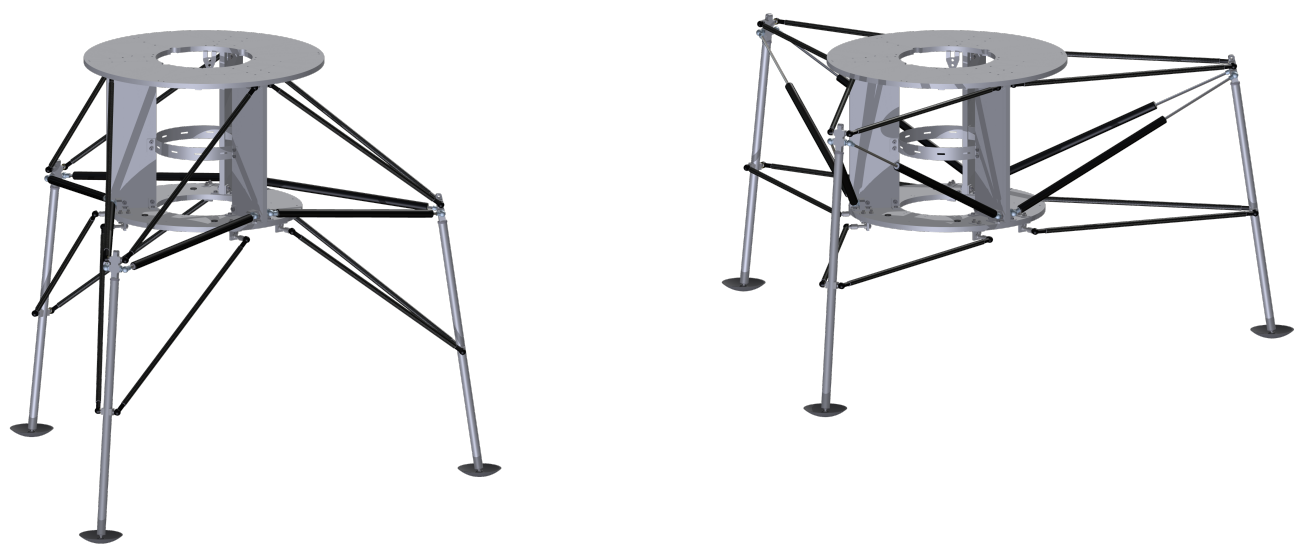

Figure 3: Landing gear system of EAGLE.

maneuvers ${ }^{\mathrm{b}}$ Finally, there is a dedicated Reaction Control System, implemented as a cold-gas thruster system.

${ }^{\mathrm{b}}$ Like a launch vehicle the roll axis points upwards in the main flight direction

$$
5 \text { of } 23
$$


Jet engine and fueling system

The jet engine generates the main thrust to act against the gravitational forces and accelerate the vehicle. Its maximal nominal static thrust is around $400 \mathrm{~N}$ at sea level at which the one-stage-radial compressor (and the one-stage turbine) rotates with approximately $100000 \mathrm{RPM}$. The only command necessary to control the engine is the pump voltage. It is issued to the Electronic Control Unit (ECU) which provides the commanded voltage to the fuel pump. In return to the command the ECU provides housekeeping data for monitoring the complex jet engine system. The whole engine and fueling system is depicted in Fig. 4 Centrally within

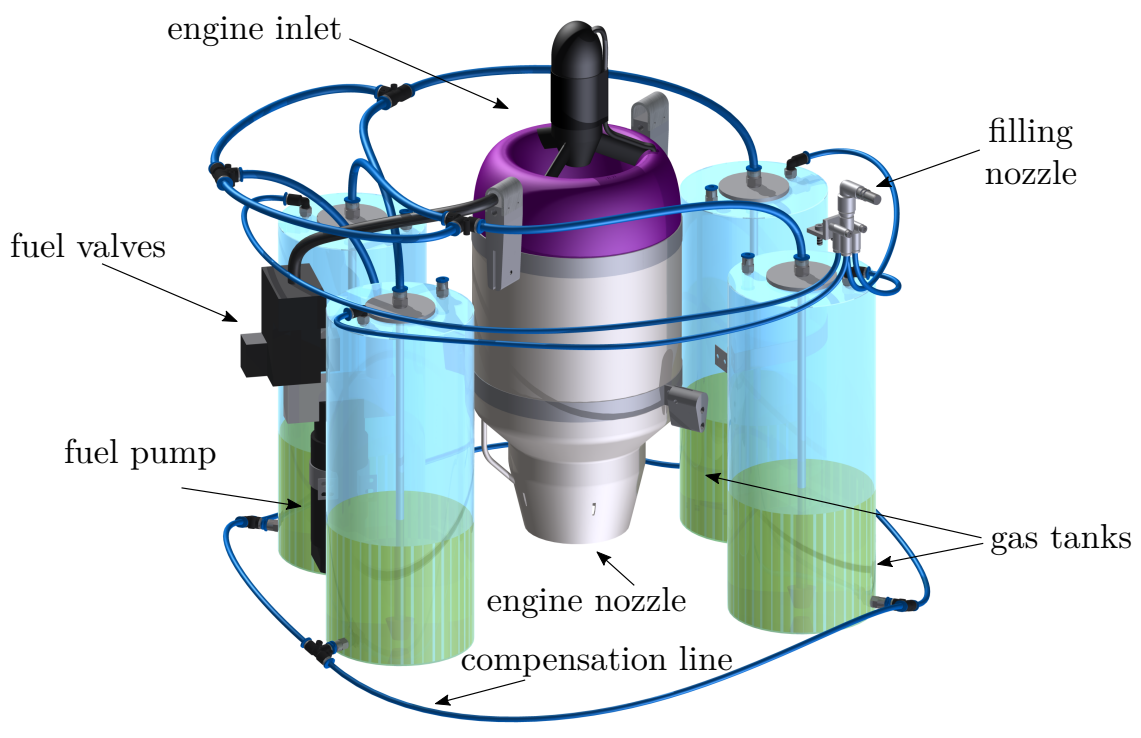

Figure 4: EAGLE's main engine and fueling system.

the setup the compact engine is shown with the inlet on the upper end and the exhaust nozzle on the lower end. It is surrounded by four 1.81 kerosene tanks that are all interconnected in order to allow equalizing their filling level.

\section{Thrust Vector Control System}

For controlling the attitude around EAGLE's pitch and yaw axes a TVC system is placed on the lower side of the main structure.

Figure 5 shows the main components that constitute the system. Each control motor rotates a shaft that

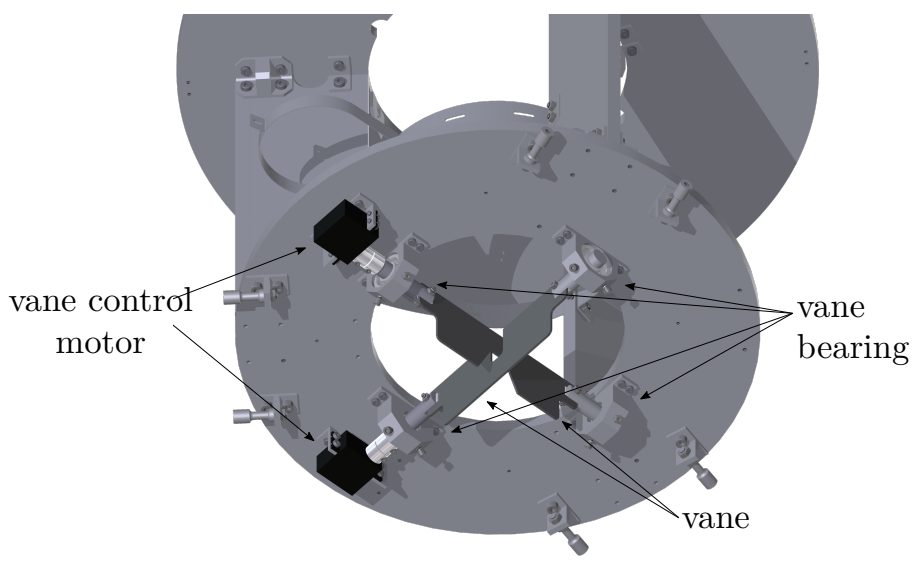

Figure 5: Thrust Vector Control system of EAGLE. 
is connected to a vane. The controllable deflection angle for each vane is about $\pm 12^{\circ}$. Higher angles are not possible due to the "v"-shaped cutouts of the vanes where the perpendicular axes cross. Each vane is supported by two ceramic ball bearings which can handle the high temperature of the jet engine's exhaust stream. The effectiveness of the system is highly depending on the main thrust. Tests with a thrust of about $270 \mathrm{~N}$ showed lateral forces of about $\pm 30 \mathrm{~N}$ at maximum deflection angle of the vanes.

Roll Control system

Figure 6 depicts the Reaction Control System used for roll control mounted on the EAGLE structure.

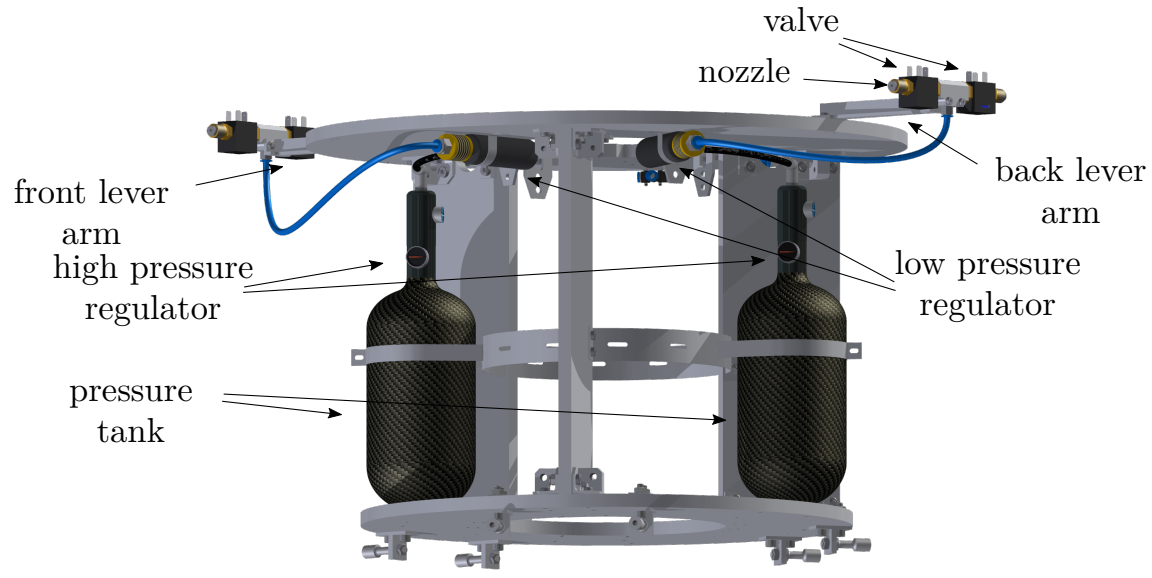

Figure 6: Cold-gas based roll control system.

The actuators that generate the control forces are connected to the front and back lever arm and consist of electro-magnetic on/off valves and a nozzles. The valves are fed with pressurized air by a hose connected to the pressure regulators.

The tanks hold 300 bar at 1.11 when full. The pressure is reduced by the two-staged system down to 12.5 bar. The nozzle is attached to the valve with a design optimized for a nominal control thrust of $4 \mathrm{~N}$.

\section{B. Avionics and Software}

The available avionics on board of EAGLE are drawn into a simple schematic in Fig. 7 The central element is the On-board computer ( $\mathrm{OBC}$ ) which has (indirectly) access to all available sensors and actuators. Depending on the electrical interface of a device it is either connected directly to the $\mathrm{OBC}$, or the data stream is routed over an Interface Board that provides access to low-level electrical interfaces (SPI, ${ }^{2} \mathrm{C}, \mathrm{PWM}$, etc.)

Communication with the ground station is guaranteed via two different wireless links on two different frequency bands. In the case of a disrupted WiFi link, a low bandwidth interface to EAGLE is still available, e. g., commanding emergency landings or monitoring the most important housekeeping data is still possible over XBee.

The OBC runs the real-time operating system QNX which executes the on-board software including the Guidance, Navigation, and Control algorithms. The main software system is built from a MATLAB/Simulink model. Simulink Coder generates "C" code from this model that is cross-compiled for the OBC. This enables a rapid prototyping functionality making it possible to make quick software changes in between tests and directly using developed and tested Simulink algorithms without the necessity of porting the algorithm to the target platform.

A real-time interface transmits the Telemetry/Telecommand (TM/TC) data between EAGLE and the ground station computer. This interface serves the two wireless interfaces that are present on EAGLE, transparently providing the telemetry data to the user from both communication channels. Additional telemetry or command parameters can be simply defined and set-up to include, e. g., new sensors, or debugging information, which is automatically logged, and time-tagged, on board and within the ground station with the rest of the telemetry. Post-processing of the logged data is established via a conversion to a MATLAB typical data file format. 


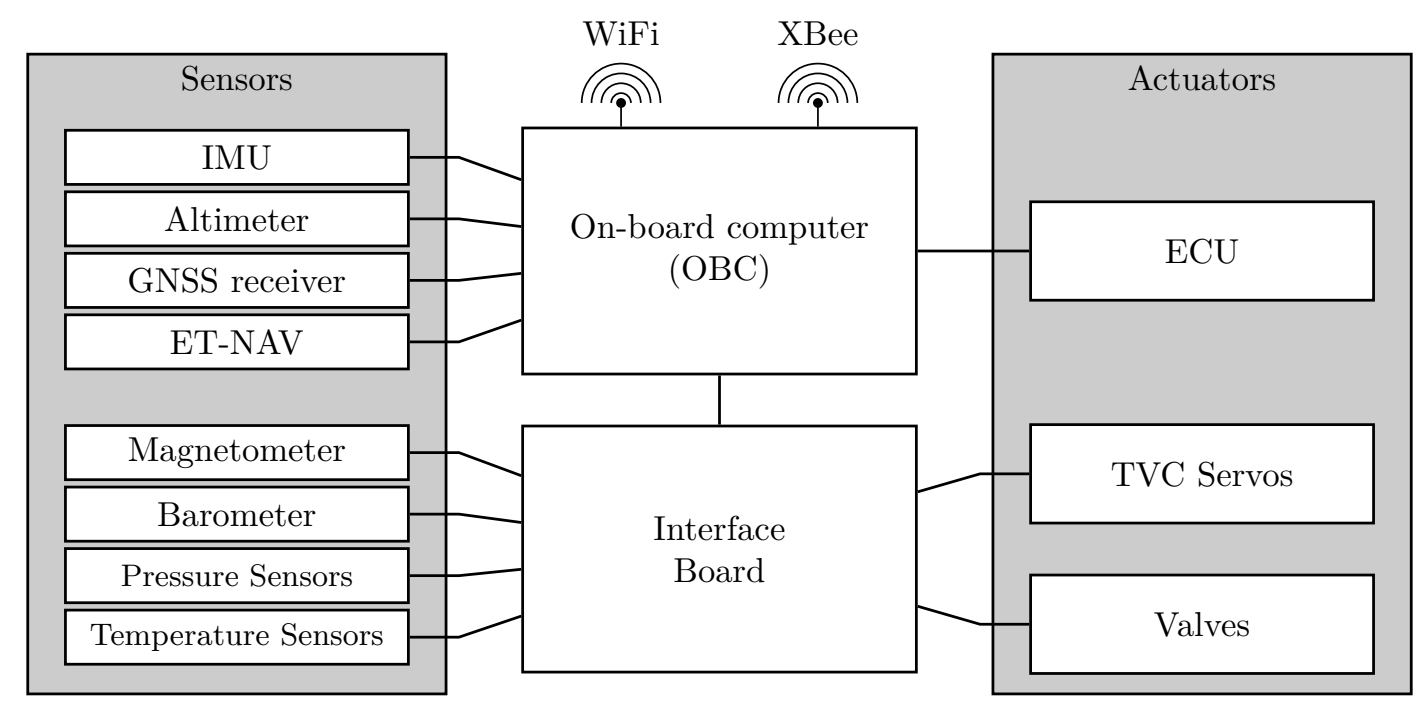

Figure 7: Avionics overview of EAGLE.

\section{System Identification}

This section briefly describes the main identification activities carried out for EAGLE. They can be divided into:

- Identification of Thrust magnitude

- Identification of Thrust vectoring

In the former case we are interested to determine the value of the thrust force $\mathbf{f}_{t h r}$ that will be generated for a given engine command. The latter deals with the determination of the lateral forces generated by deflecting the vanes at a given thrust level. The identification activities were necessary to identify the torques and the forces that the controller can generate with the available actuators, and how accurate and reproducible they are. Moreover, it was necessary to map these forces and torques to the commands that the actuators actually receive. For this purpose some experimental tests were carried out, and a series of identification strategies was conducted.

A. Identification of Thrust magnitude

In EAGLE a jet engine provides the main thrust force $\mathbf{f}_{t h r}$, which is assumed to be generated along the $x$ axis of the body reference frame $\mathrm{B}$.

$$
\mathbf{f}_{t h r}=\left\|\mathbf{f}_{t h r}\right\| \mathbf{e}_{x}^{\mathrm{B}}
$$

This reference frame has its origin in the Center of Mass (CoM) of EAGLE and is aligned with the mechanical system $\mathrm{M}$. The $y$ and $z$ axes define the pitch and yaw axes, and are controlled through the rotation of the two TVC vanes. The $\mathrm{B}$ and $\mathrm{M}$ reference frames are depicted in Fig. 8

The thrust force can be modulated by changing the pump voltage level, which represents the command signal for the thrust. However, a more intuitive command control, called Engine Power Settings (EPS), was used.

The EPS can be related to the voltage level through a linear relationship of the form

$$
\mathrm{EPS}=a_{v} V+b_{v}
$$

where $a_{v}$ and $b_{v}$ are constant values chosen such that EPS is equal to 0 when the engine is at idle, and to 100 when the engine is in full-thrust regime. The final objective of this identification activity is therefore the construction of the relationship between EPS and $\left\|\mathbf{f}_{t h r}\right\|$, which eventually coincides in identifying the physical relationship between the pump voltage and the thrust magnitude. The identification was carried

$$
8 \text { of } 23
$$




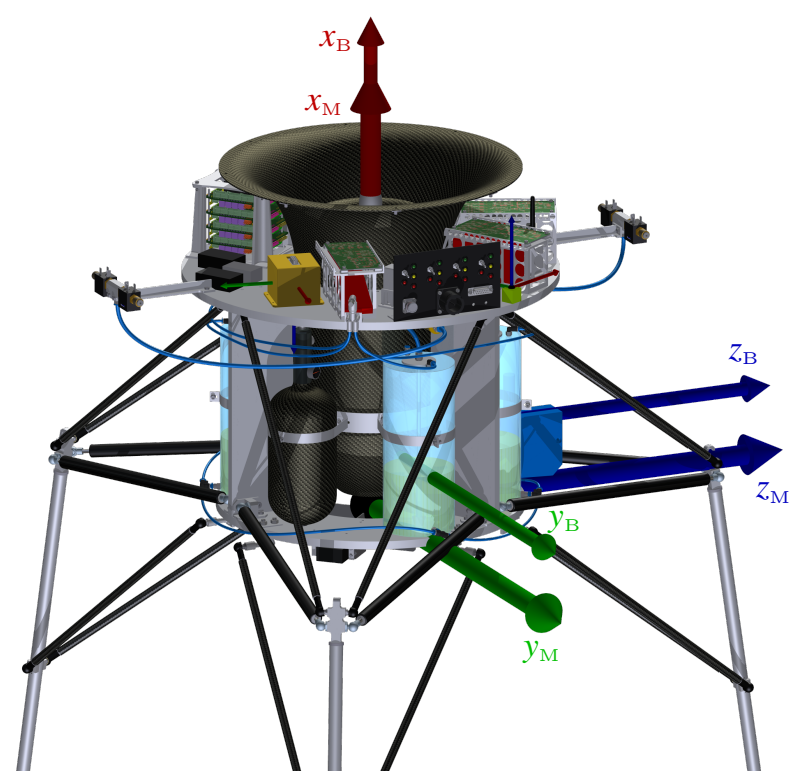

Figure 8: Mechanical reference system $\mathrm{M}$ and the body reference system B of EAGLE.

out on a fixed testbench with EAGLE being mounted horizontally on a force-torque sensor to reduce the effects of an engine close to ground 10

Several values of EPS were provided and the generated force measured. The collected data was then fit into a polynomial,

$$
\left\|\mathbf{f}_{t h r}\right\|=a_{3} \operatorname{EPS}^{3}+a_{2} \operatorname{EPS}^{2}+a_{1} \operatorname{EPS}+a_{0}
$$

The overlapping of this model with some of the measurements is shown in Fig. 9 The static model

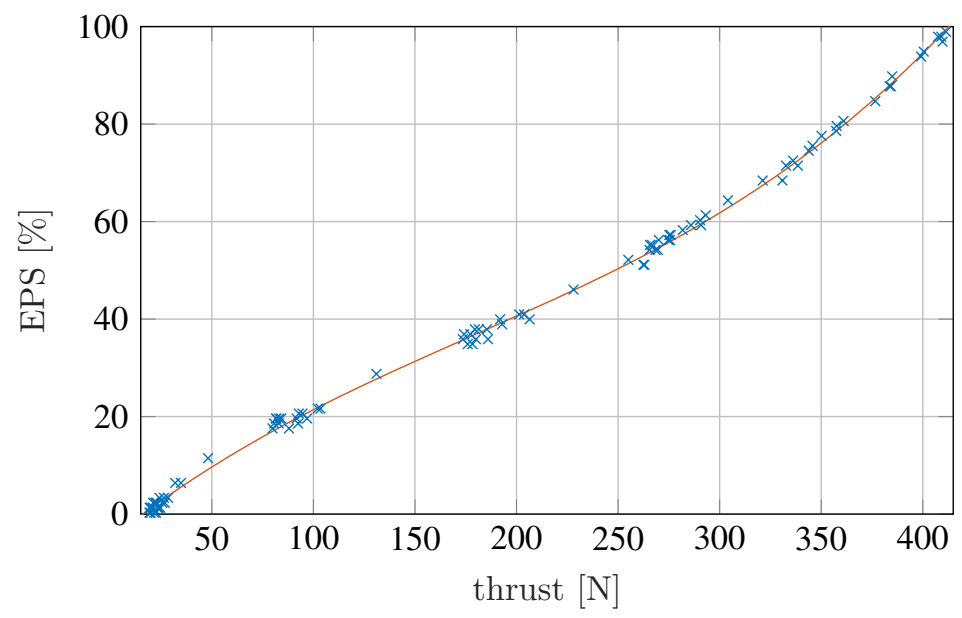

Figure 9: Thrust-EPS identification: the red line represents the model, while the red crosses some of the measurements taken in a given test session.

was identified over multiple engine runs on different days to gain confidence on the results, and was then compared with experimental data. An example of the results obtained is depicted in Fig. 10

The bottom plot shows the recorded commands, which were sent to the engine during the tests (orange line), and were reproduced during the identification phase (blue line). The top plot shows the corresponding measurements (orange line) and the predictions made by the identified model (blue line). It is possible to 

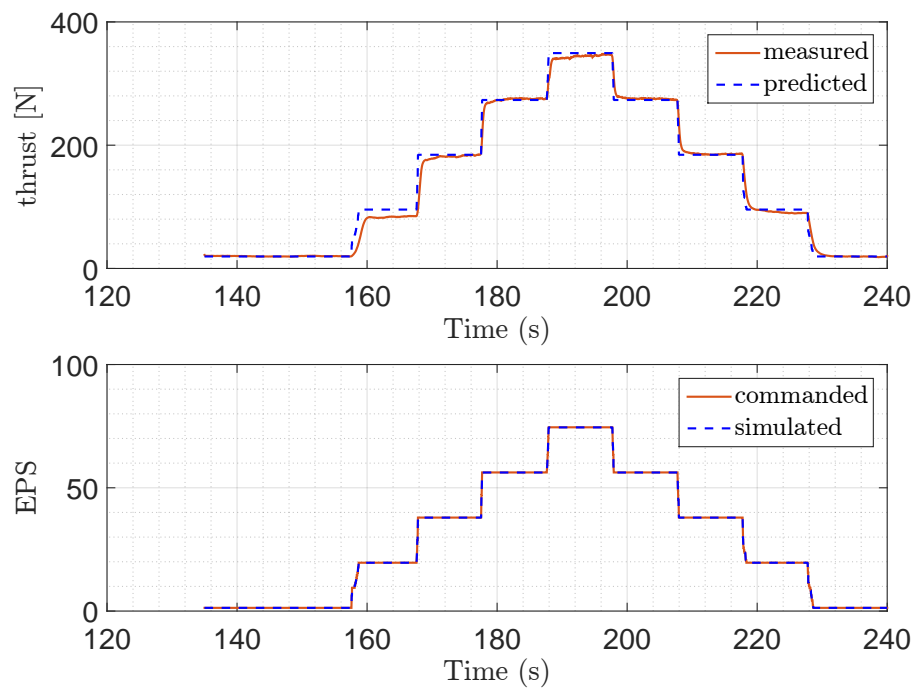

Figure 10: Thrust-magnitude comparison of model prediction with experimental data.

observe that the model matches the measurement data quite well.

\section{B. Identification of Thrust vectoring}

The second part of the identification process dealt with the thrust vectoring. In fact, the thrust $\mathbf{f}_{t h r}$ is mainly responsible for counteracting the gravity, but parts of the main thrust can be deflected for attitude thrust-vector control. The resulting force of the deflected main thrust is $\mathbf{f}_{\mathrm{TVC}}$. Having known the Center of Pressure (CoP) of the vanes by construction (Fig. 5), and the CoM by measurements, it is possible to estimate the lever arm $\mathbf{I}_{\mathrm{TVC}}$, and therefore to predict the torques computed by the TVC system.

The TVC force $\mathbf{f}_{\mathrm{TVC}}$ can be transformed into a torque $\mathbf{t}_{\mathrm{TVC}}$ by using

$$
\mathbf{t}_{\mathrm{TVC}}=\mathbf{l}_{\mathrm{TVC}} \times \mathbf{f}_{\mathrm{TVC}}
$$

The components of the force $\mathbf{f}_{\mathrm{TVC}}$ can be modulated by rotating the vanes to deflect the thrust force $\mathbf{f}_{t h r}$. These vanes are actuated via servos $v_{0}$ and $v_{1}$, which, together with the EPS represent the actual commands for the thrust vector control. Therefore an identification procedure was needed to determine the mapping between commands and generated forces

$$
\left\{\begin{array}{c}
\mathrm{EPS} \\
v_{0} \\
v_{1}
\end{array}\right\} \longleftrightarrow \mathbf{f}_{\mathrm{TVC}}
$$

Note that these maps are built on the basis of the previous knowledge acquired in terms of EPS-Thrust. This second part of identification aimed at determining the side-forces generated by the TVC system at a given thrust level. The tests were performed under three different thrust levels. The middle thrust is an assumed level necessary to hover EAGLE, counteracting the gravitational force, and an upper and lower level: $\pm 30 \mathrm{~N}$ around hover thrust.

On each of theses three thrust values a sequence of vane deflections was commanded focusing more on small angles, making the identification more accurate in the region which is assumed important for stabilizing EAGLE. These identification maps were decoupled for each of the vanes, that is the full thrustvector identification was reduced to determine two separate maps, which provide the following relationships

$$
\left\{\begin{array}{c}
\left\|\mathbf{f}_{t h r}\right\| \\
v_{0}
\end{array}\right\} \longleftrightarrow \mathbf{f}_{z, \mathrm{TVC}}, \quad\left\{\begin{array}{c}
\left\|\mathbf{f}_{t h r}\right\| \\
v_{1}
\end{array}\right\} \longleftrightarrow \mathbf{f}_{y, \mathrm{TVC}}
$$




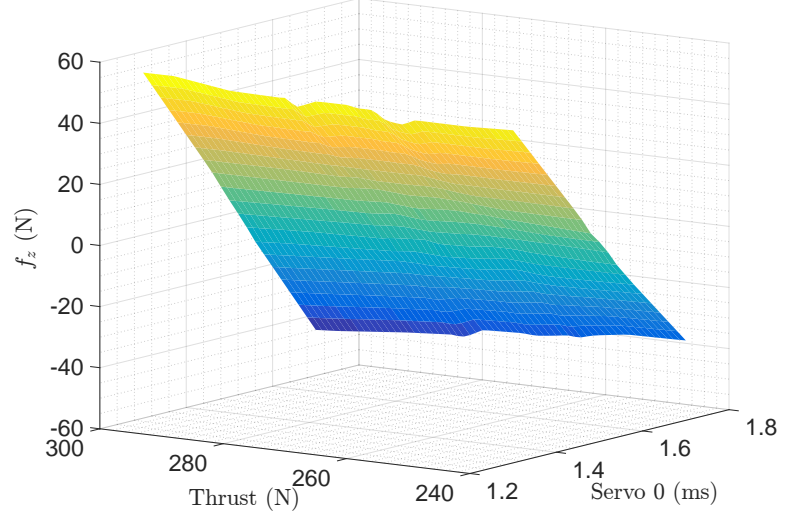

(a) Map linking thrust level, vane deflection $v_{0}$ and side- (b) Map linking thrust level, vane deflection $v_{1}$ and sideforce $f_{z}$

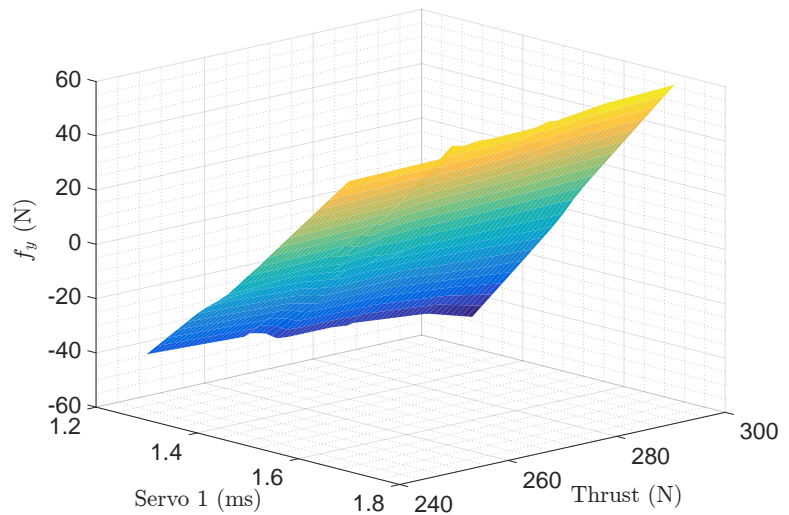

force $f_{y}$

Figure 11: Identified EAGLE vanes - forces maps.

The maps obtained are depicted in Figs. 11a and 11b The results show that decoupled forces can be assumed within the region of small deflection angles. Moreover, the maps show an almost linear behavior, which is consistent with the expectations. As in the previous case, validation activities w.r.t. the measurements have been performed. An example of these validation activities is shown in Fig. 12, The same commands used to generate the measurements (orange lines) were fed into the identified model (blue lines). The predictions were then overlapped with the measurements. For all the experiments there is a good agreement between predicted and measured side forces. The error was in most of the cases less than $0.2 \mathrm{~N}$.

The determination of the thrust magnitude and the thrust direction for a given set of commands EPS, $v_{0}, v_{1}$ concludes the identification phase. It was therefore possible to move to the following phase, i.e., the design the controller.
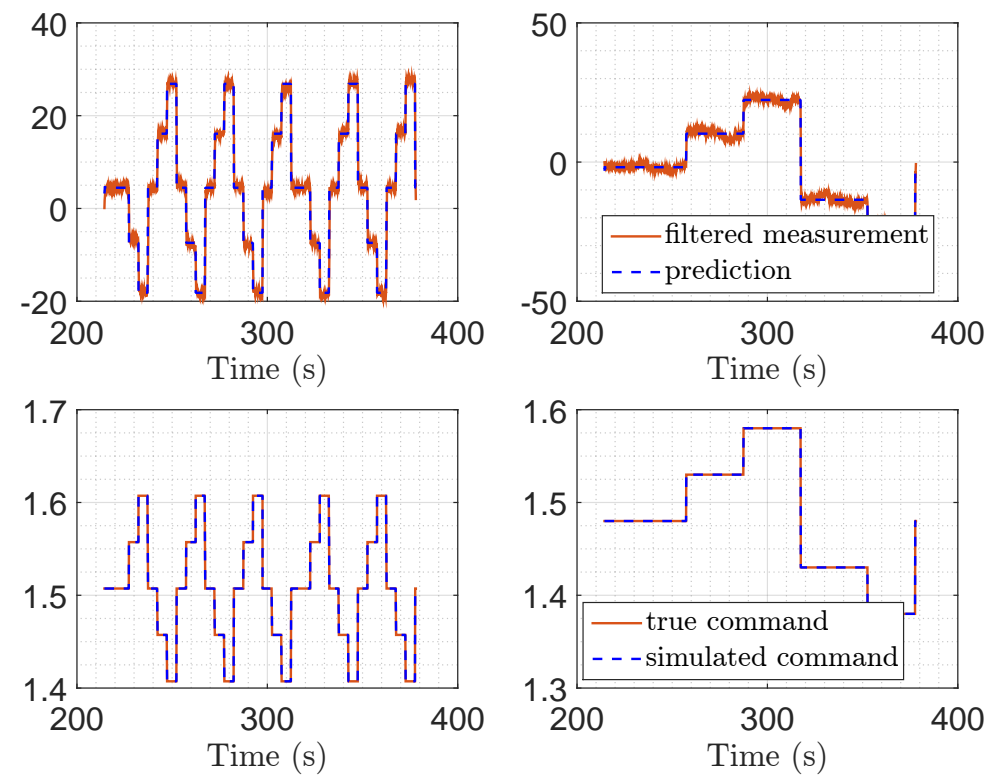

Figure 12: Thrust-vector comparison of model prediction with experimental data. 


\section{Control}

Despite the activities of system identification carried out, the control system of EAGLE has to cope with a complex, nonlinear scenario. Uncertainties as well as further unmodeled disturbances like wind and ground effects can strongly affect the results. For EAGLE a divide-and-conquer strategy based on a separation of the controllers has been employed. An overview of the control scheme adopted is visible in Fig. 13

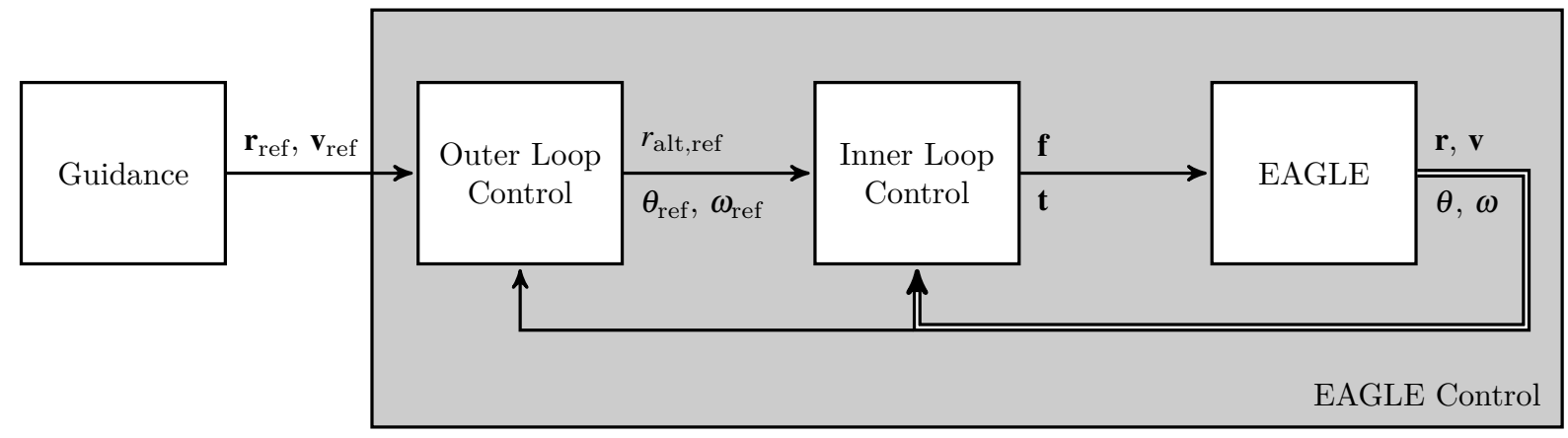

Figure 13: EAGLE control scheme.

We can distinguish a guidance module, an outer loop, which is responsible for the position, and an inner loop, responsible for the attitude. Note that throughout this work a very simple guidance, represented by an altitude step command, has been used, while in future we plan to compute optimal guidance solutions in real time (e.g., by using pseudospectral convex optimization ${ }^{11}$ ). The generated forces $\mathbf{f}$ and torques $\mathbf{t}$ will determine the rotational and translational dynamics of EAGLE, which are described by a mass-varying 6-DOF model,

$$
\begin{aligned}
\dot{\mathbf{r}} & =\mathbf{v} \\
\dot{\mathbf{v}} & =\frac{\mathbf{f}}{m}+\mathbf{g}-\frac{\dot{m}}{m} \mathbf{v} \\
\dot{\theta} & =\omega \\
\dot{\omega} & =\mathbf{I}^{-1}[\mathbf{t}-\omega \times \mathbf{I} \cdot \omega]
\end{aligned}
$$

where $\mathbf{r}$ and $\mathbf{v}$ are the position and the velocity of EAGLE expressed in the $N E D$ reference frame, defined as having the $\hat{D}$ axis pointing towards the center of the Earth, the $\hat{N}$ axis towards the North, and $\hat{E}$, pointing towards East, is defined as

$$
\hat{E}=\hat{D} \times \hat{N}
$$

The $N E D$ reference frame is depicted in Fig. 14

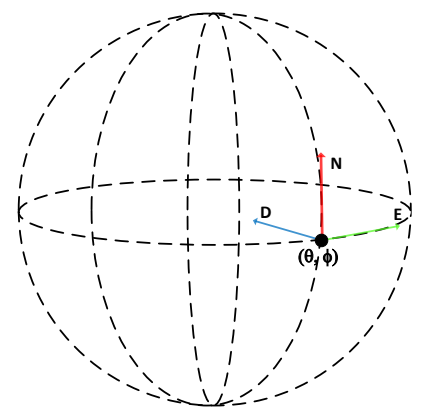

Figure 14: NED Reference frame definition at a given longitude $\theta$ and latitude $\phi$.

The attitude is parametrized by using the Euler angles $\theta$ and the angular rate $\omega$, expressed in body reference frame; $m$ and $\mathbf{I}$ are the mass, and the inertia of EAGLE. Note that while the mass variation was taken into account, the inertia was assumed constant, in order to keep the model as simple as possible. 
With these definition it is possible to design the controller for EAGLE. The roll control has been independently developed for practical reasons: since it is based on the use of cold-gas system, it could be tested independently during the assembly of the engine, and therefore was developed and validated first, $\frac{12}{12}$ and later on integrated within the thrust-vector control strategy.

We refer to thrust-vector control as the control strategy aimed at stabilizing the attitude while having EAGLE hovering at a given distance from ground. In fact, the inner loop has two separate controllers, the thrust-vector controller, which is in charge of stabilizing the pitch and yaw axes, and the altitude controller. It would be therefore improper to define it as attitude control since also one of the translational components is involved. This development direction comes from the possibility to test the attitude stabilization and the hovering capabilities before having the outer loop (i.e., the position control) in place, guaranteeing therefore the required flexibility and modularity in the development and the prototyping of EAGLE. Finally, we refer to position control as the control activity aimed at tracking a given planar position, that is the motion in the plane orthogonal to the hovering direction. As suggested by the name, the roll control module stabilizes the motion around the roll axis. A more in-depth scheme of the control strategy is shown in Fig. 15.

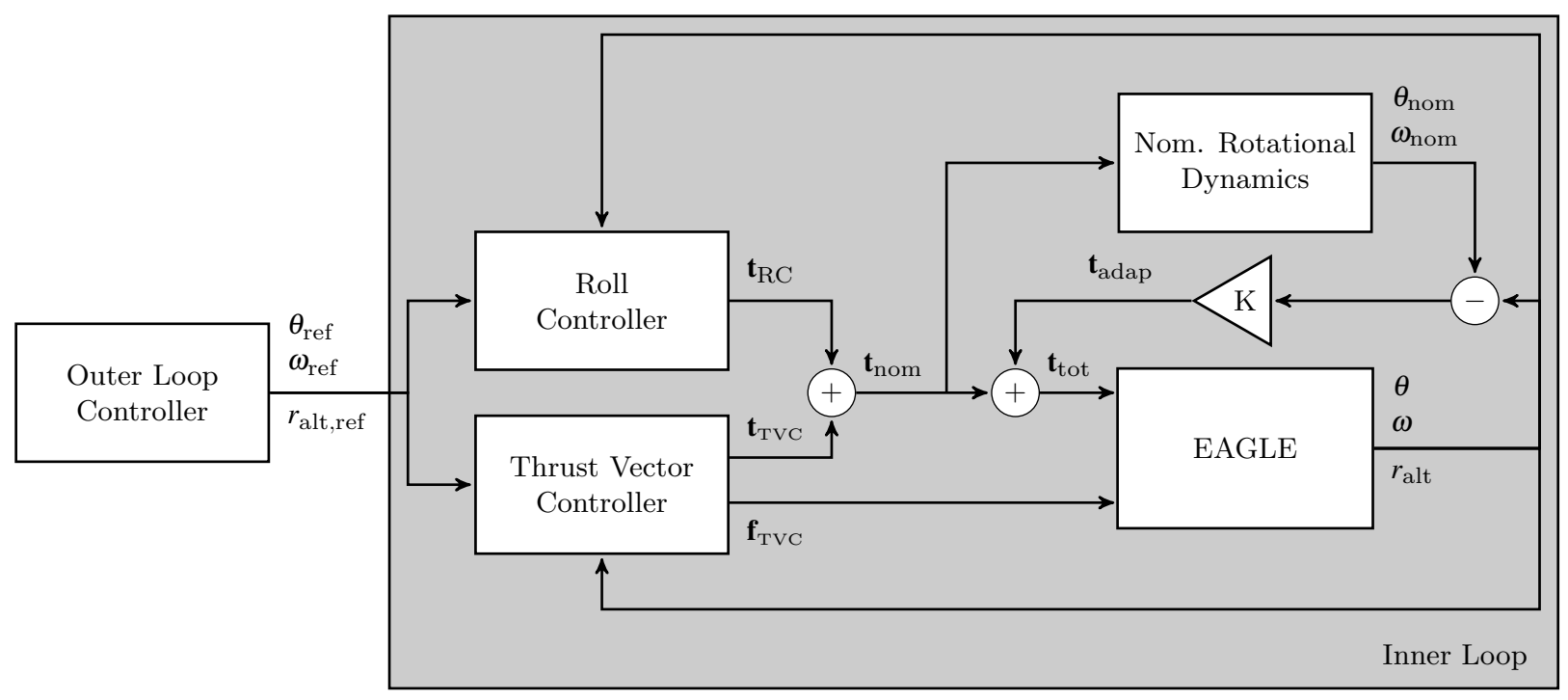

Figure 15: Detailed EAGLE control system scheme.

The outer loop computes the reference Euler angle $\theta_{\text {ref }}$ and the reference altitude $r_{a l t}$. The thrust-vector control and the roll control compute the torque required to control the attitude. At the same time, the thrust for ensuring the hovering of EAGLE is computed. This term is augmented with an adaptive term, to make the controller able to mimic the behavior of the nominal system even in presence of significant uncertainties. Sections $\mathrm{A}, \mathrm{B}$ and $\mathrm{C}$ give more information about the control structure.

\section{A. Roll Control}

Roll control has to satisfy two needs: first, it has to keep the angular rate close to 0 . The second mode refers to keep a specific roll angle. Therefore, we can distinguish three modes:

- angular rate control, to ensure uncoupled motion;

- minimum-time angle control, to reach a specified angle in the shortest possible time;

- angle control, which aims at keeping the roll angle at a specific value;

A cold-gas system is used for this task. The cold-gas system is controlled through PWM, and therefore, an on-off switching logic has been implemented. The angular rate control aims at keeping the angular rate close to zero. To included hysteresis and dead-zone in the controller, a Schmitt Trigger $\frac{13}{13}$ in the command of valves is implemented. For the minimum-time roll control a minimum-time algorithm is used ${ }^{14}$ It is known that is algorithm provides good results in achieving the desired angle, but it is not so efficient in keeping 
the set angle. Therefore, once that a given threshold is reached, the angle-control mode is triggered. The output generated is a torque able to control the roll motion of EAGLE.

$$
\mathbf{t}_{R C}=\left[\begin{array}{c}
t_{x} \\
0 \\
0
\end{array}\right]
$$

More details about these specific algorithms can be found in the previous work of Leite Filho et al 12

\section{B. Thrust Vector Control}

The thrust-vector control strategy includes the stabilization of the altitude $r_{x}$, as well as of the first two Euler angles $\theta_{v}$, and $\theta_{z}$, which are representative of pitch and yaw. The control strategy is based on sliding-mode theory ${ }^{[\sqrt[5]{5}}$ For each of the variables to track a corresponding sliding surface $s_{i}, i=[x, y, z]$ is designed. The chosen sliding surfaces are of the form

$$
s_{p} \triangleq \dot{e}_{p}+\lambda_{p} e_{p} \triangleq \dot{p}-\dot{p}_{r e f}+\lambda_{p}\left(p-p_{r e f}\right), \quad p \in[x, y, z]
$$

where $e_{p}$ represents the error we want to minimize, and the terms $\lambda_{p}, p \in[x, y, z]$ are the closed-loop bandwidths of the system. From the sliding surfaces it is possible to derive the nonlinear control signals which drive the corresponding Lyapunov functions to zero. If we look at the first two of Eqs. (7), and we take the first component we can write

$$
\ddot{r}_{x}=A_{x}+B_{x} f_{x}
$$

with

$$
\begin{aligned}
A_{x} & =-\frac{\dot{m}}{m} v_{x}-g_{x} \\
B_{x} & =\frac{1}{m}
\end{aligned}
$$

If we instead focus on the last two of Eqs. (7), by keeping in mind that $\ddot{\theta}=\dot{\omega}$, we can rewrite them in affine form as

$$
\ddot{\theta}=\mathbf{A}_{\theta}+\mathbf{B}_{\theta} \mathbf{t}
$$

where

$$
\begin{aligned}
& \mathbf{A}_{\theta}=-\mathbf{I}^{-1}[\omega \times \mathbf{I} \cdot \omega] \\
& \mathbf{B}_{\theta}=\mathbf{I}^{-1}
\end{aligned}
$$

In order to ensure that the system is globally stable, that is

$$
\begin{aligned}
& x \longrightarrow x_{\text {ref }} \\
& \theta \longrightarrow \theta_{\text {ref }}
\end{aligned}
$$

a Lyapunov function, which is a measure of the attitude error, has to be defined. A Lyapunov function has to satisfy the following properties:

$$
V(s) \text { Lyapunov function } \Longleftrightarrow\left\{\begin{array}{c}
\lim _{s \rightarrow \infty} V(s) \rightarrow \infty \\
V(s)>0 \quad \forall s \neq 0 \\
\dot{V}(s)<0 \quad \forall s \neq 0
\end{array}\right.
$$

In other words, the function has to be globally positive definite, while having its derivative globally negative definite. Moreover, it has to be radially unbounded. It is possible to show that if such a function exists, the variable $s$ of the corresponding dynamical system will go to 0 , and the system will be asymptotically stable. The function chosen in this case is

$$
V\left(s_{p}\right)=\frac{1}{2} s_{p}^{2}, \quad p \in[x, y, z]
$$

It is trivial to observe that the first two conditions are easily satisfied. If we develop the third one, we can write

$$
\dot{V}\left(s_{p}\right)=\dot{s}_{p} s_{p}<0
$$


The key idea of sliding mode control is to assign a convergence rate to the derivative of Lyapunov function, that is

$$
\dot{V}\left(s_{p}\right)=\dot{s} s_{p}=-k_{p}|s|, \quad k_{p}>0, p \in[x, y, z]
$$

where the gain $k_{p}$ is a design parameter, also called nonlinear gain of the sliding mode controller. For $s_{p} \neq 0$ we can further manipulate Eq. 19 as

$$
\dot{s_{p}}=-k_{p} \operatorname{sign}\left(s_{p}\right), \quad p \in[x, y, z]
$$

where the function sign is defined as

$$
\operatorname{sign}(s)=\left\{\begin{array}{rr}
1, & s \geq 0 \\
-1, & s<0
\end{array}\right.
$$

Note that to avoid chattering ${ }^{5}$ the sign function is replaced by a saturation function, defined as

$$
\operatorname{sat}(s)=\left\{\begin{array}{cc}
1, & s \geq \delta \\
-1, & s \leq-\delta \\
s / \delta, & |s|<\delta
\end{array}\right.
$$

where $\delta$ is a small value, which identifies the region of linearity of the controller. The use of the saturation function represents a trade-off as the robustness, theoretically ensured by the presence of the sign function, is partially reduced to avoid chattering and get a smoother control action. Note that more advanced techniques, such as the use of high-order SMC5 5 can be employed to avoid chattering. However, in this phase of development the purpose was to keep the architecture as simple as possible. Eq. (18) can be further developed by taking Eqs. (10) and (14) into account. In fact, we can write

$$
\ddot{p}-\ddot{p}_{r e f}+\lambda_{p}\left(\dot{p}-\dot{p}_{r e f}\right)=-k_{p} \text { sat }\left[\dot{p}-\dot{p}_{r e f}+\lambda_{p}\left(p-p_{r e f}\right)\right], \quad p \in[x, y, z]
$$

If we replace Eqs. 111) and (13) within Eq. 23) we get

$$
\overbrace{\left[\begin{array}{c}
A_{x} \\
\mathbf{A}_{\theta, y, z}
\end{array}\right]}^{\mathbf{A}}+\overbrace{\left[\begin{array}{cc}
B_{x} & \mathbf{O}_{1 \times 2} \\
\mathbf{O}_{2 \times 1} & \mathbf{B}_{\theta, y, z}
\end{array}\right]}^{\mathbf{B}}+\overbrace{\left[\begin{array}{c}
f_{x} \\
\mathbf{t}_{y, z}
\end{array}\right]}^{\mathbf{u}}+\overbrace{\left[\begin{array}{c}
-\ddot{x}_{r e f} \\
-\ddot{\theta}_{r e f, y, z}
\end{array}\right]+\left[\begin{array}{c}
\lambda_{x} e_{x}+\dot{e}_{x} \\
\lambda_{\theta, y, z} \mathbf{e}_{\theta, y, z}+\dot{\mathbf{e}}_{\theta, y, z}
\end{array}\right]}^{\mathbf{K}}=-\overbrace{\left[\begin{array}{c}
k_{x} \operatorname{sign} s_{x} \\
k_{\theta} \operatorname{sign} s_{\theta, y, z}
\end{array}\right]}^{\mathbf{E}}
$$

With these definitions, the affine form represented by Eq. 24) can be solved with respect to the control $\mathbf{u}$,

$$
\mathbf{u}=-\mathbf{B}^{-1}(\mathbf{K}+\mathbf{A}+\Lambda)
$$

which represents the sliding mode control which maps the sliding surfaces $s_{x}, s_{y}$, and $s_{z}$ to the force $f_{x}$, and to the torques $t_{y}$ and $t_{z}$, which guarantee the stability of the system. Note that the control law represented by Eq. 25 is applicable only when the matrix $\mathbf{B}$ is non-singular, that is, only when the condition

$$
\operatorname{det}(\mathbf{B}) \neq 0
$$

holds. An analysis of the terms which appear in the matrix $\mathbf{B}$ show that these conditions are satisfied if and only if

$$
\left\|\mathbf{f}_{\mathbf{t h r}}\right\| \neq 0
$$

which means, as it can be easily intuited, that the system can only be controlled if a thrust force is generated. Therefore, Eq. 27] is satisfied during the entire flight time.

The torque can be augmented by taking the mismatch between real plant and nominal plant into account. This is done by computing an adaptive control term, which is included in the loop.

$$
\mathbf{t}_{T V C}=\mathbf{t}_{\text {nom }}+\mathbf{t}_{\text {adap }}
$$

Given the force $f_{x}$ and the torques $t_{y}$ and $t_{z}$, we can back-calculate the vanes deflections and the EPS required for the actual control of EAGLE in virtue of the maps introduced in Sec. III.

$$
\left\{\begin{array}{c}
f_{x} \\
t_{y} \\
t_{z}
\end{array}\right\} \longleftrightarrow\left\{\begin{array}{c}
\mathrm{EPS} \\
v_{0} \\
v_{1}
\end{array}\right\}
$$

Finally, since the TVC is not able to control the roll, the dedicated roll control, defined in Eq. (9), is integrated in the feedback control law.

$$
\mathbf{t}_{\text {tot }}=\mathbf{t}_{T V C}+\mathbf{t}_{R C}
$$




\section{Position Control}

Also for the position control a sliding-mode control strategy was implemented. In this case it was necessary to define two sliding surfaces for the "in-plane" components of position $y$ and $z$. The dynamics along the axes $y$ and $z$ can be written in affine form as

$$
\begin{aligned}
& \ddot{r}_{y}=A_{y}+B_{y} f_{y} \\
& \ddot{r}_{z}=A_{z}+B_{z} f_{z}
\end{aligned}
$$

with

$$
\begin{aligned}
& A_{y}=-\frac{\dot{m}}{m} v_{y}+g_{y} \\
& A_{z}=-\frac{\dot{m}}{m} v_{z}+g_{z} \\
& B_{y}=B_{z}=\frac{1}{m}
\end{aligned}
$$

The corresponding sliding surfaces are formally defined in this case as

$$
s_{p} \triangleq \dot{e}_{p}+\lambda_{p} e_{p} \triangleq \dot{p}-\dot{p}_{r e f}+\lambda_{p}\left(p-p_{r e f}\right), \quad p \in[y, z]
$$

Note that throughout this section the $y$ and $z$ subscripts will refer to the force, position, velocity and acceleration components. Since the thrust force is determined by the inner loop the sliding surfaces $s_{y}$ and $s_{z}$ need to be linked to the two side-forces $f_{y}$ and $f_{z}$ needed for controlling the position of EAGLE. Also in this case we can manipulate the expression given in Eq. 200 and write

$$
\dot{s_{p}}=-k_{p} \operatorname{sign}\left(s_{p}\right), \quad p \in[y, z]
$$

To compute the forces we can recall Eq. (11) and plug them into Eq. (34). We get

$$
\overbrace{\left[\begin{array}{c}
A_{y} \\
A_{z}
\end{array}\right]}^{\widetilde{\mathbf{A}}}+\overbrace{\left[\begin{array}{cc}
B_{y} & 0 \\
0 & B_{z}
\end{array}\right]}^{\widetilde{\mathbf{B}}} \overbrace{\left[\begin{array}{c}
f_{y} \\
f_{z}
\end{array}\right]}^{\mathbf{f}_{y z}}+\overbrace{\left[\begin{array}{c}
-\ddot{y}_{r e f} \\
-\ddot{z}_{r e f}
\end{array}\right]+\left[\begin{array}{l}
\lambda_{y} e_{y}+\dot{e}_{y} \\
\lambda_{z} e_{z}+\dot{e}_{z}
\end{array}\right]}^{\widetilde{\Lambda}}=-\overbrace{\left[\begin{array}{c}
k_{y} \operatorname{sign} s_{y} \\
k_{z} \operatorname{sign} s_{z}
\end{array}\right]}^{\widetilde{\mathbf{k}}}
$$

and we can compute the side-force required for the control of EAGLE as

$$
\left[\begin{array}{c}
f_{y} \\
f_{z}
\end{array}\right]=\mathbf{f}_{y z}=-\widetilde{B}^{-1}(\widetilde{K}+\widetilde{A}+\widetilde{\Lambda})
$$

These components will become part of the compound force acting on the vehicle, defined as

$$
\mathbf{f}=\left[\begin{array}{l}
f_{x} \\
f_{y} \\
f_{z}
\end{array}\right]
$$

The controllability analysis in this case ensures that the system is always controllable. A deeper analysis of the side-forces shows the position controllability of EAGLE is ensured once again only if Eq. (27) holds.

Note that the force magnitude is commanded by the altitude control. Therefore the physical mechanism to generate side-forces is to rotate EAGLE, which means that the position control is ensured by rotating EAGLE such that the projection of the thrust force is equal to the forces computed through Eq. (36). These components will be transformed into a corresponding reference quaternion $\mathbf{q}_{B, \text { ref }}$, which will be transformed into a set of reference Euler angles $\theta_{\text {ref }}$ which feed the inner loop. To compute the quaternion we can define

a unit vector representing the force direction in case of no error on position and attitude, that is, when the thrust is aligned with the $x$ axis.

$$
\widehat{\mathbf{f}}_{\text {nom }}=\left[\begin{array}{l}
1 \\
0 \\
0
\end{array}\right]
$$


The real, normalized force required for the position control while hovering is

$$
\widehat{\mathbf{f}}_{\text {real }}=\|\mathbf{f}\|^{-1}\left[\begin{array}{c}
f_{x} \\
f_{y} \\
f_{z}
\end{array}\right]
$$

The angle $\phi$ between $\widehat{\mathbf{f}}_{\text {nom }}$ and $\widehat{\mathbf{f}}_{\text {real }}$ and the corresponding rotation axis $\widehat{e}_{\phi}$ are

$$
\begin{aligned}
\cos \phi & =\left(\widehat{\mathbf{f}}_{\text {nom }} \cdot \widehat{\mathbf{f}}_{\text {real }}\right) \\
\widehat{e}_{\phi} & =\widehat{\mathbf{f}}_{\text {real }} \times \widehat{\mathbf{f}}_{\text {nom }}
\end{aligned}
$$

It is finally possible to obtain the corresponding reference quaternion $q_{\mathrm{B}, \text { ref }}$ as

$$
\mathbf{q}_{\mathrm{B}, r e f}=\left[\begin{array}{r}
e_{\phi, x} \sin (\phi / 2) \\
e_{\phi, y} \sin (\phi / 2) \\
e_{\phi, z} \sin (\phi / 2) \\
\cos (\phi / 2)
\end{array}\right]
$$

This transformation is depicted in Fig. 16, where the nominal force, the real force and the rotation axis are represented in the nominal B reference frame. The projection of $\widehat{f}_{\text {real }}$ on the axes $y$ and $z$ provide the side-forces required to control EAGLE. Note that the angle $\phi$ has been enlarged for a better visualization of the components of $\mathbf{f}_{\text {real }}$.

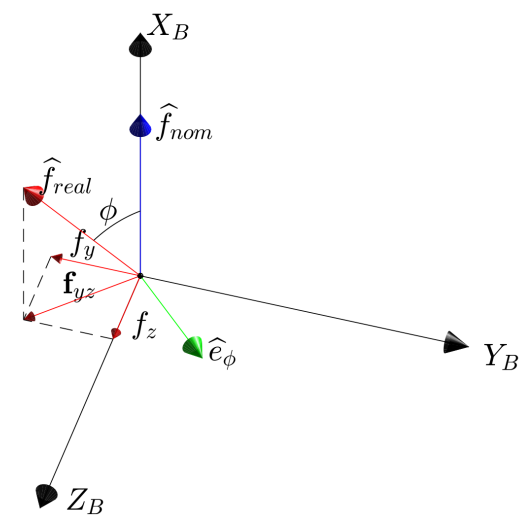

Figure 16: Determination of reference quaternion based on the side-forces required to control the position of EAGLE.

Note that we implicitly assume that the reference quaternion $\mathbf{q}_{\mathrm{B}, \text { ref }}$ provides small rotations (i.e., $\phi \leq 5$ deg), such that the reduction of the $x$ component of the force can be neglected, that is

$$
f_{x} \cos (\phi) \approx f_{x}
$$

This condition can be ensured by proper tuning of the SMC, and is practically verified both in the simulations and in the tests performed.

\section{Nominal Simulation}

First, we implemented a proper simulator for EAGLE. The simulator tested a complete take-off - hovering - landing sequence, and had the navigation subsystem in the loop, to ensure high-fidelity of the results. The objective was to hover for about $30 \mathrm{~s}$ at $1 \mathrm{~m}$, while keeping the position and the attitude stable.

Fig. 17 shows the position, velocity of EAGLE, together with the attitude, represented in terms of roll, pitch and yaw, and the control modes, which manage the different flight phases. 
The hovering command was sent at $t=110$ to allow for a proper initialization of the navigation solution. The control mode 0 indicates that no thrust is generated. When the mode 1 is triggered the engine is turned on and the thrust level is increased linearly to a level that is slightly lower than the one required to hover. Control mode 3 triggers a thrust level larger than the EAGLE's weight, and when the lift-off is detected (mode 4) the full closed-loop logic is triggered. This mode is hold for about 30 seconds. After this time interval a landing command is sent to EAGLE, which reduces its thrust level and therefore its altitude. Through the entire flight sequence it is possible to observe that the altitude is correctly kept, together with the altitude and the position. Note that the planar position is less accurate than the altitude, in virtue of the presence of the laser altimeter. The accuracy of the attitude is in the order of 0.2 degrees. The controls (Fig. 18) are consistent. The thrust level converges to the weight of EAGLE, and the vanes deflection are in the order of 2 degrees. Finally, the sliding surfaces $s_{x}, s_{y}$ and $s_{z}$ converge to 0 , as expected.
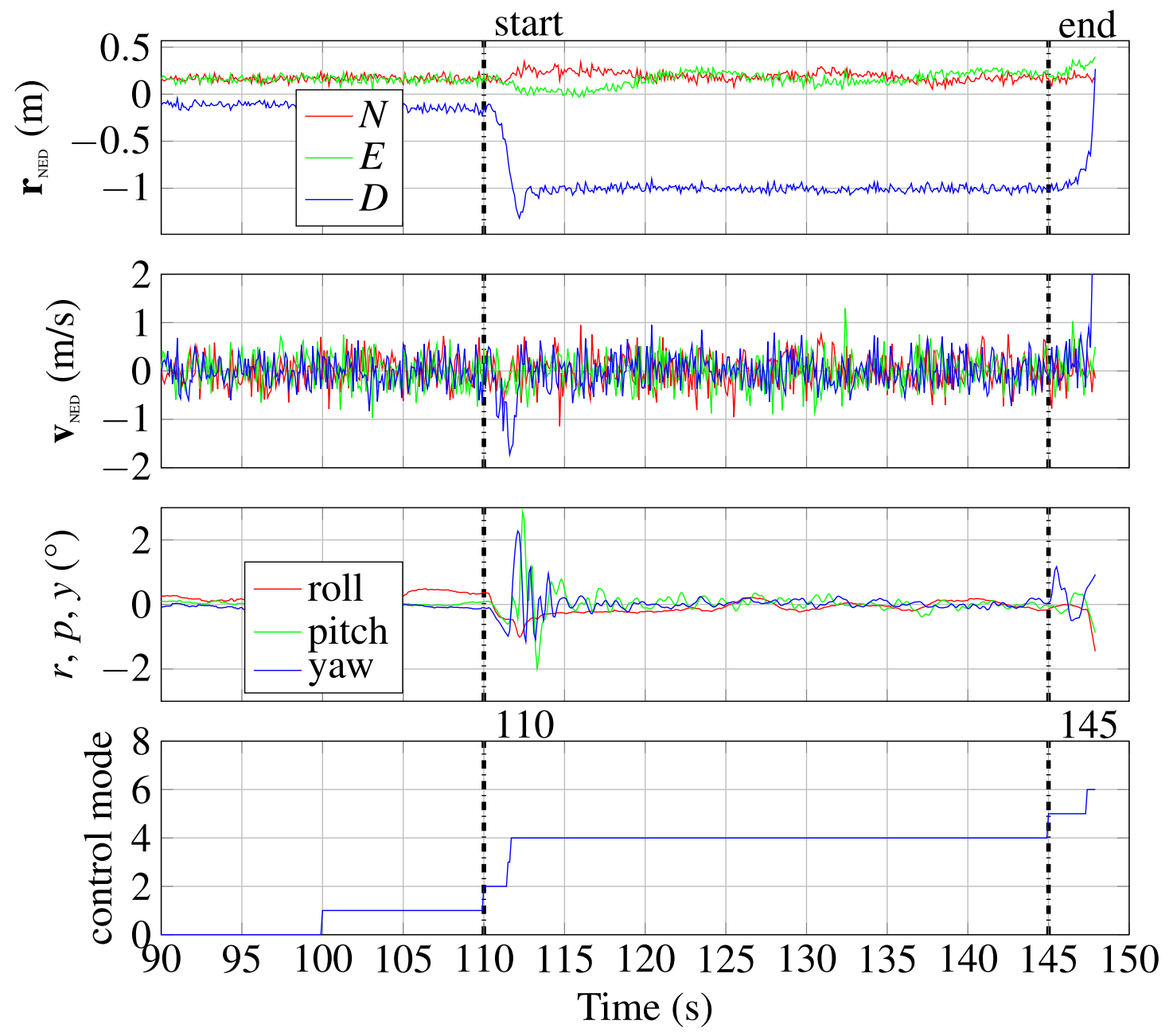

Figure 17: From the top: EAGLE position, (velocity) in NED reference frame, attitude, and control mode (d).

In terms of control the results are depicted in Fig. 18

\section{Tethered flight results}

\section{A. NEST}

NEST is a facility built at the Institute of Space Systems in 2016. This test facility was designed to safely handle EAGLE during the first hovering tests, but also for later tests when EAGLE's software or hardware 

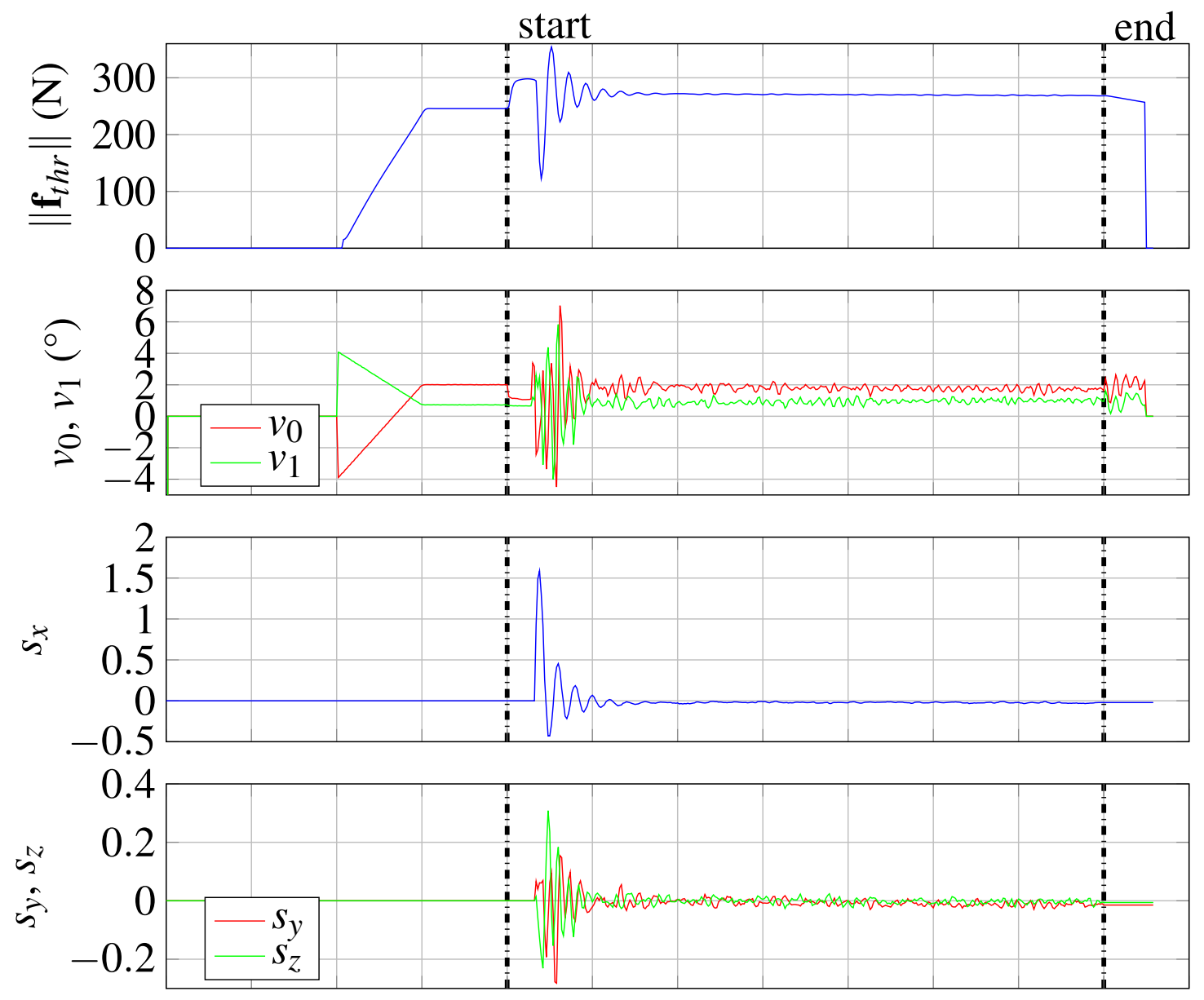

Time (s)

Figure 18: From the top: EAGLE vanes deflections, thrust magnitude, sliding surfaces for the altitude, and attitude.

was modified and a safe operation could not be guaranteed prior to the test. It is a triangular structure equipped with an upper part able to sustain EAGLE. Figure 19 shows the setup with only a single tether to the top of the vehicle which is used if the GNC system is already reliable, and was the configuration used for the experiments showed here.

Within this test setup we proved the feasibility of hovering EAGLE within a small confined space applying a high standard for the safety of EAGLE as well as personnel and observers. At the same time it allows a quick access to the lander for conducting consecutive tests with a short turn-around-time.

Depending on the maturity of the overall system, different ways of tethering EAGLE are possible. The picture shows the setup with only a single tether to the top of the vehicle which is used if the GNC system is already reliable. The tether is connected to a rubber band dampening jerks during lift-off and landing, and in unforeseen situations, when the tether is stretched.

Three separate tests were conducted to prove the functioning of the several controller components for altitude (B), attitude (C), and position (D).

\section{B. Altitude controller}

This section shows the first performance test of the sliding-mode altitude controller. The performance of this controller is expected to improve in the future, but the results show that the current maturity level is sufficient to stabilize the altitude of EAGLE. Due to the fact that the space where EAGLE can move freely 


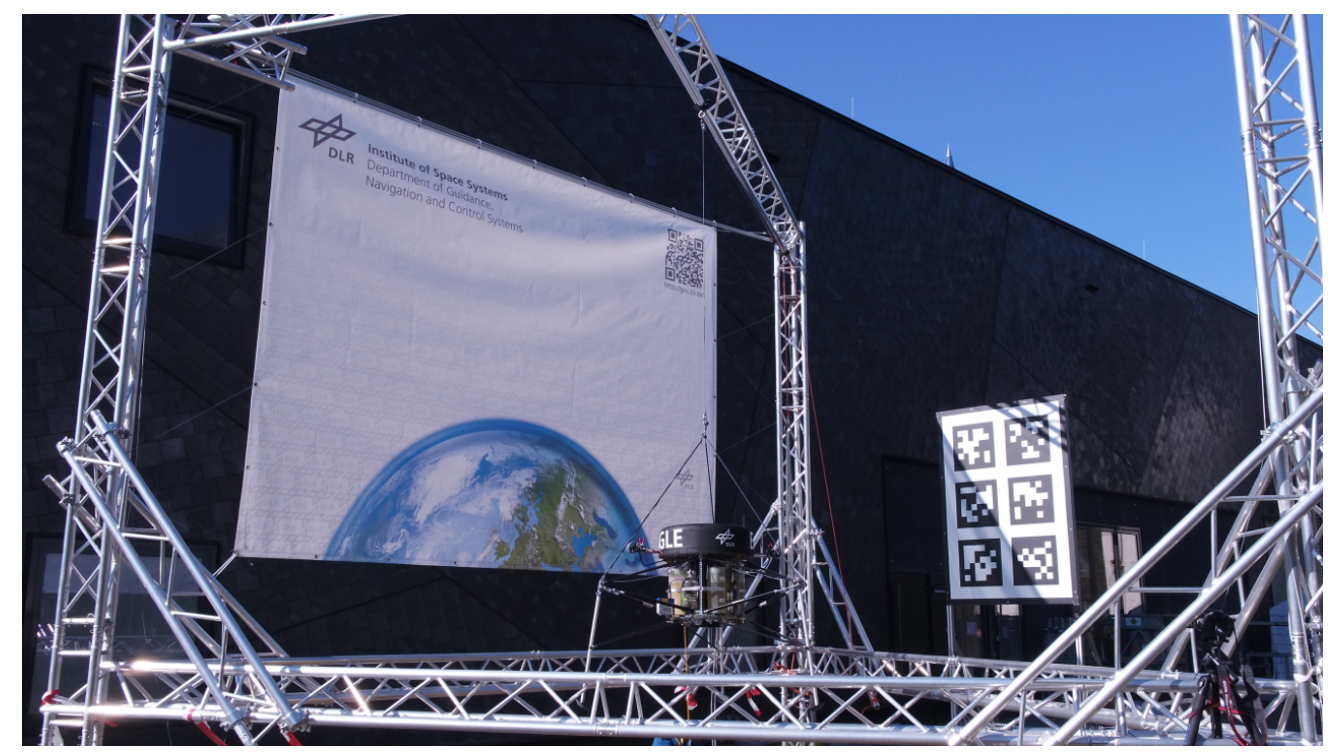

Figure 19: DLR NEST tethered flight test facility.

within the tethered flight facility is restricted, the altitude controller is tested within a range of $0.5 \mathrm{~m}$ to $1.2 \mathrm{~m}$ only. The dynamic behavior below $0.5 \mathrm{~m}$ is heavily disturbed by the tether which is pulled up by the rubber band. The upper limit is defined by the NEST setup, and the navigation solution.

Figures $20 \mathrm{a}$ and $20 \mathrm{~b}$ shows these limits captured during the test. These limits are also highlighted in Fig. 21.

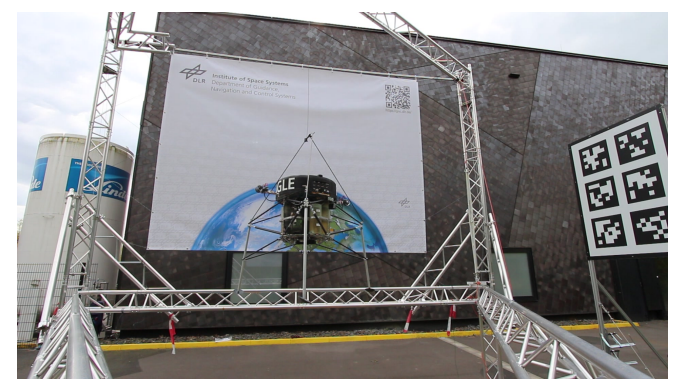

(a) Lower bound of the clearance

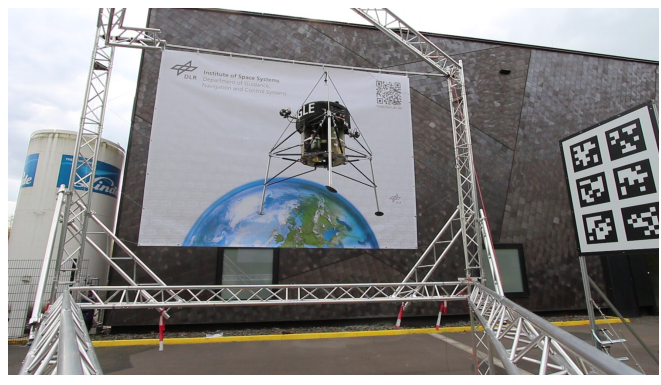

(b) Upper bound of the clearance

Figure 20: The altitude bounds for EAGLE during the sliding-mode altitude controller test.

Within these limits EAGLE was commanded to different set-points for the altitude to evaluate the performance and control quality of the new controller. Figure 22 shows the data recorded during the test.

The upper plot shows the commanded and measured altitude over the test cycle, the negative sign comes from the down direction from the chosen North East Down (NED) reference frame. The climb and descent cycles, and stationary flights follow the set-point commanded by the ground station. The control error (depicted in the lower plot) clearly shows a general constant offset that is smaller than $20 \mathrm{~cm}$. The spikes on the other hand result from the dynamics of EAGLE. When a new set-point was issued the error instantaneously grows and in the following seconds the relatively slow engine thrusts up (or down) to maneuver EAGLE to the new position reducing the error.

There are probably multiple reasons for constant deviation. First, the sliding-mode tuning was not very aggressive, and was tuned to resemble a PD controller for gaining confidence during the tests. Moreover, for the initial test campaign it was decided to suppress the integral part of the controller, to avoid problems coming from a saturating integrator of a sub-optimally tuned controller. Furthermore, the magnitude of the control deviation is largely dominated by the feed-forward part, that roughly acts as gravity compensation. 


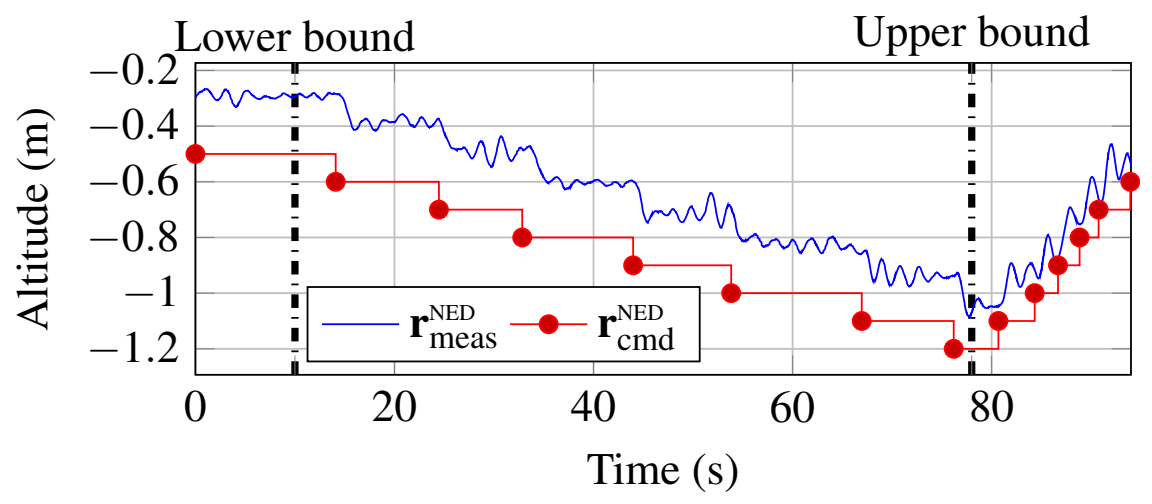

Figure 21: Altitude control - Upper and lower bounds for the tests.
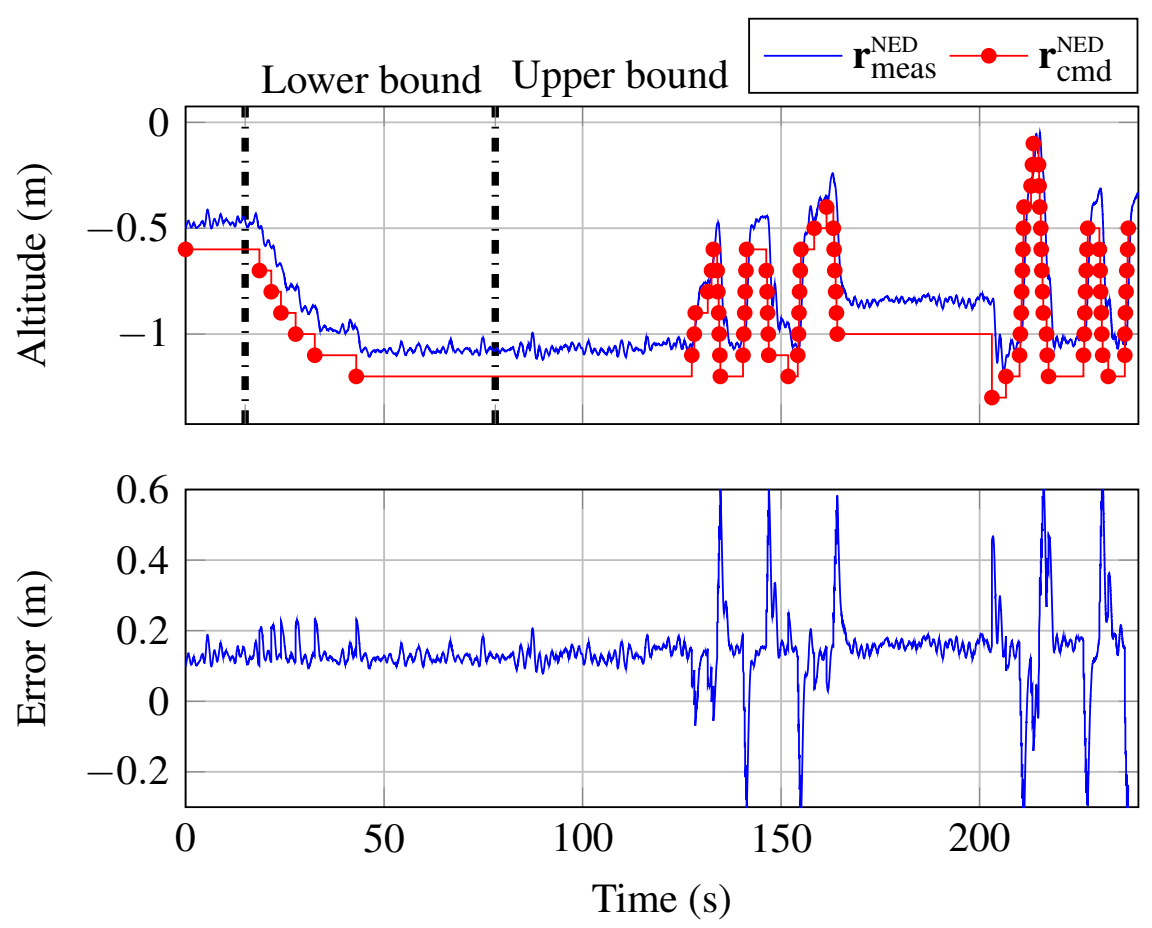

Figure 22: From the top: EAGLE altitude, and altitude error.

This feed-forward contribution assumes a well identified engine thrust and a known mass, that is changing over time. Both requirements are only known with large uncertainties, resulting in the given deviation from the set-point. The performance will increase in the future by refining the tuning of the parameters, and allowing for a stronger action of the nonlinear part of the controller. Eventually, better estimates for the physical characteristics of EAGLE, like Mass, Center of Mass, Inertia (MCI), actuator identification, etc., will also improve the overall controller performance.

\section{Attitude controller}

Further flights were conducted between July and September 2017 to test several aspects of EAGLE. Among these the SMC-based attitude controller. Results for one of the flights are depicted in Fig. 23 In the first plot on top we can see the pitch and yaw evolution. During the entire flight the oscillations are within $\pm 2^{\circ}$. Note that the roll evolution has been omitted here as its controller has a separate design, which 
is not directly connected to the SMC. Further details about the roll controller can be found in! 10 In the second plot of Fig. 23 we show the vanes deflections required to control EAGLE. The deflections are in the range $[-3,+5]^{\circ}$, well within the capabilities of the actuators $\left( \pm 12^{\circ}\right)$. For what regards the sliding surfaces, depicted at the bottom we can observe that the sliding surfaces are stable around the origin, although not completely converging. This is mainly due to the tuning of the controller, which during this first phase of flight test was not too aggressive.

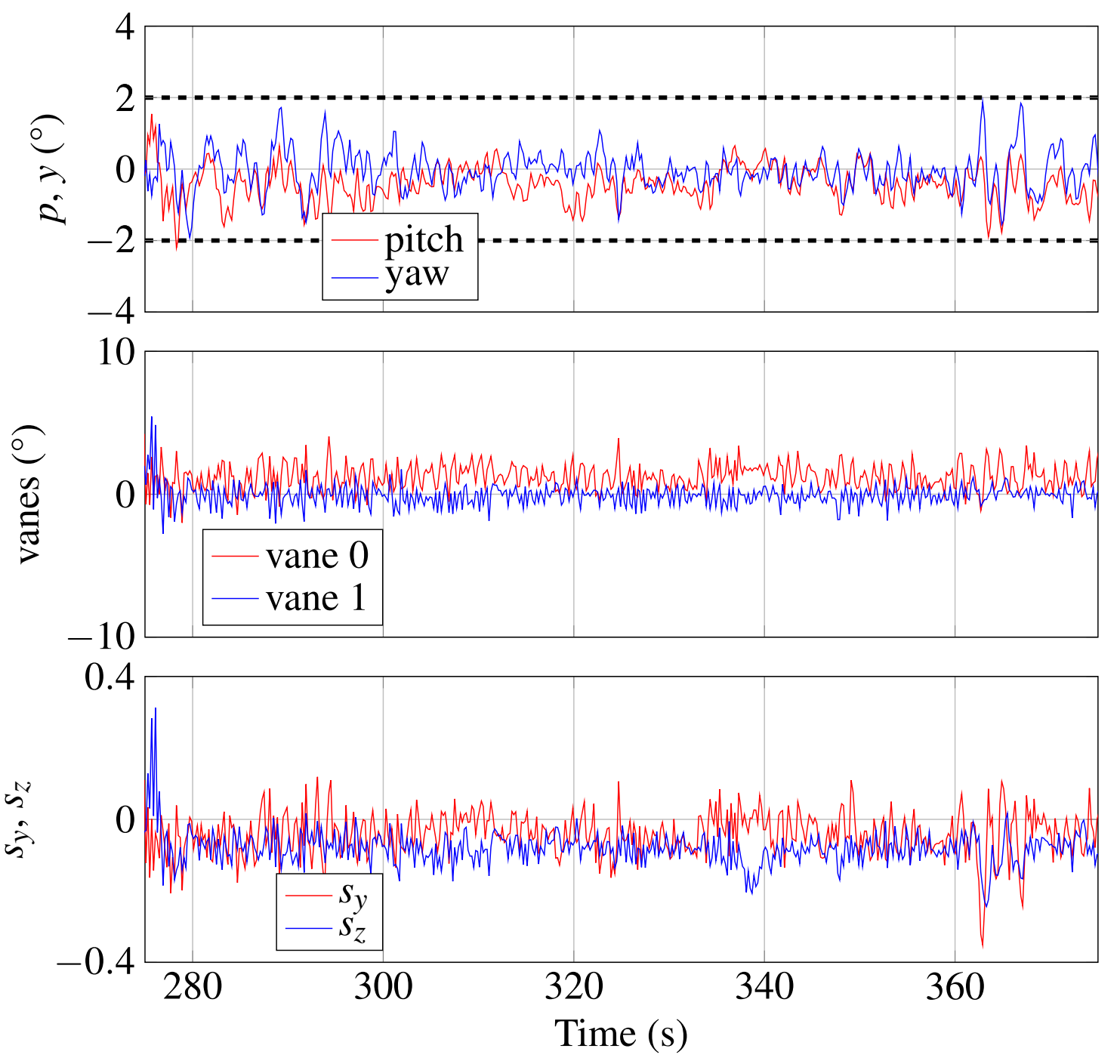

Figure 23: From the top: EAGLE attitude, vanes deflection, and corresponding sliding surfaces.

\section{Position controller}

The outer loop was also tested. The corresponding results are depicted in Fig. 24. The first subfigure shows the in-plane components of the position. Oscillations remain within $1 \mathrm{~m}$ from the origin. The corresponding forces and the sliding surfaces are depicted in the second and third plots. Also in this case a preliminary soft tuning was employed, leading to forces within $10 \mathrm{~N}$ and a value of the sliding surfaces of about \pm 0.2 . Also in this case a more aggressive tuning will be implemented during the next tests to assess the impact on the performance in terms of position errors. 

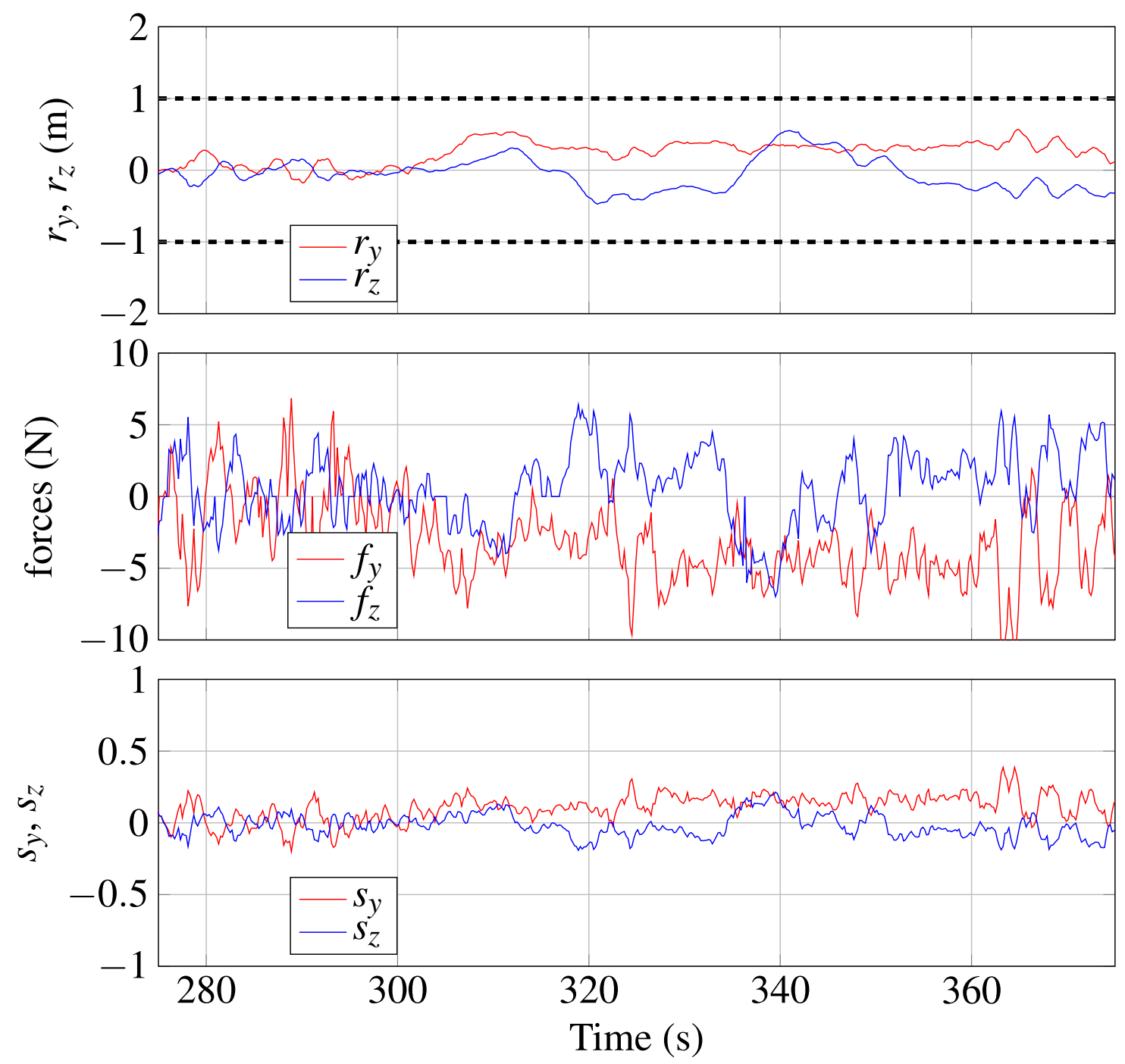

Figure 24: From the top: EAGLE position in-plane components, forces, and corresponding sliding surfaces.

\section{Conclusions and outlook}

In this paper the design of the control strategy for EAGLE has been described. An overall system overview has been given, together with the identification activities carried over to characterize the vehicle and the actuators devoted to control it. We presented some first simulations, together with the preliminary flight test results, which show that the control strategy is suitable for a complex, constrained scenario involving VTOL vehicles. To the best of our knowledge this is the first time that an intrinsic nonlinear control strategy, specifically sliding mode theory, is used and demonstrated on an experimental flight vehicle, showing that these technologies are earning the maturity level expected for real missions.

For what regards EAGLE we demonstrated the hovering capability. The immediate next steps are launching from ground and landing on ground. Additionally, other settings and algorithms for guidance and control can be easily implemented and tested. A further possibility is the testing of pseudospectral convex optimization for the real-time generation of guidance solutions. Another major step is conducting free flights. For that a few more logistical and administrative steps have to be completed in order to get access to a test range with a restricted air space and to receive the corresponding flight permissions. Of course, the highest possible confidence in the system has to be achieved with more tethered flight tests.

In parallel two studies at Deutsches Zentrums für Luft- und Raumfahrt (DLR) are going on which explore 
different options to extend the existing system. The first study focuses on creating a larger version "EAGLE XL" which is using a larger jet engine as the main thruster, e. g. an engine with a nominal thrust of $1500 \mathrm{~N}$. Since the larger engine is more fuel efficient and many of the subsystems do not scale one-to-one with the size of the engine a larger version of EAGLE would allow either for more payload or more fuel mass enabling more flight time and a larger flight envelope.

A second study focuses on replacing the jet engine with a set of green mono-propellant rocket engines. This configuration would go beyond the pure testing of GNC and would allow to gain test results and handling experience with a propulsion system which is very similar to planetary landers or returning first stages with a powered landing. Finally, the test vehicle EAGLE is available to the community as part of joint research projects and studies.

\section{Acknowledgments}

The development and implementation of EAGLE is an internal project of the Institute of Space Systems of the German Aerospace Center (DLR). The final phase was supported as part of the project "Upper Stage Attitude Control Design Facility (USACDF)" under subcontract of Airbus Defense and Space Germany and the European Space Agency.

\section{References}

${ }^{1}$ Gu, C., Vernis, P., J.F. Jourdas, J., Hachelef, N., Heynen, S., Poirrier, T., Schwientek, A., and Boudillet, O., "HOMER PROJECT: Development, validation, qualification and flight test results of the autonomous flight control of the test vehicle," 9th International ESA Conference on Guidance, Navigation \& Control Systems, June 2014.

${ }^{2} \mathrm{Gu}$, C., Heynen, S., and Poirrier, T., "HOMER Take-off: A Review," dSPACE Magazine, Vol. 2015, No. 2, June 2015, pp. $32-35$.

${ }^{3}$ Perruquetti, W., Sliding Mode Control in Engineering, Marcel Dekker Inc, 2002.

${ }^{4}$ Fernandez, B. and Hedrick, K. J., "Control of Multivariable Non-Linear Systems by the Sliding Mode Method," Int. J. Control, Vol. 46, No. 3, pp-1019-1040, 1987.

${ }^{5}$ Shtessel, Y. B., Edwards, C., Fridman, L., and Levant, A., Sliding Mode Control and Observation, Birkhäuser, 2013.

${ }^{6} \mathrm{Xu}, \mathrm{H} .$, Mirmirani, M. D., and Ioannou, P. A., "Adaptive Sliding Mode Control Design for a Hypersonic Flight Vehicle," Journal of Guidance, Control, and Dynamics, Vol. 27, No. 5, Sept. 2004, pp. 829-838.

${ }^{7}$ Furfaro, R., Cersosimo, D., and Wibben, D. R., "Asteroid Precision Landing via Multiple Sliding Surfaces Guidance Techniques," Journal of Guidance, Control, and Dynamics, Vol. 36, No. 4, July 2013, pp. 1075-1092.

${ }^{8}$ Furfaro, R. and Wibben, D., "Mars Atmospheric Entry Guidance via Multiple Sliding Surface Guidance for Reference Trajectory Tracking," Guidance, Navigation, and Control and Co-located Conferences, American Institute of Aeronautics and Astronautics, Aug. 2012.

${ }^{9}$ Sagliano, M., Mooij, E., and Theil, S., "Adaptive Disturbance-Based High-Order Sliding-Mode Control for HypersonicEntry Vehicles," Journal of Guidance, Control, and Dynamics, Vol. 40, No. 3, March 2017, pp. 521-536.

${ }^{10}$ Dumke, M., Sagliano, M., Saranrittichai, P., Trigo, G. F., and S., T., "EAGLE - Environment For Autonomous GNC Landing Experiments," 2017.

${ }^{11}$ Sagliano, M., "Pseudospectral Convex Optimization for Powered Descent and Landing," Journal of Guidance, Control, and Dynamics, 2018.

${ }^{12}$ Leite Filho, W. C., Dumke, M., and S., T., "Control System Design of the Lander Demonstrator EAGLE," EUCASS, 2015.

${ }^{13}$ Wie, B., Space Vehicle Dynamics and Control, AIAA Education Series, American Institute of Aeronautics and Astronautics, Inc., 2008.

${ }^{14}$ Kirk, D. E., Optimal Control Theory - An Introduction, Dover, 1970. 